Algebraic 85 Geometric Topology

Volume 2 (2002) 465-497

Published: 22 June 2002

ATG

\title{
A new invariant on hyperbolic Dehn surgery space
}

\author{
James G. Dowty
}

\begin{abstract}
In this paper we define a new invariant of the incomplete hyperbolic structures on a 1-cusped finite volume hyperbolic 3-manifold $M$, called the ortholength invariant. We show that away from a (possibly empty) subvariety of excluded values this invariant both locally parameterises equivalence classes of hyperbolic structures and is a complete invariant of the Dehn fillings of $M$ which admit a hyperbolic structure. We also give an explicit formula for the ortholength invariant in terms of the traces of the holonomies of certain loops in $M$. Conjecturally this new invariant is intimately related to the boundary of the hyperbolic Dehn surgery space of $M$.
\end{abstract}

AMS Classification 57M50; 57M27

Keywords Hyperbolic cone-manifolds, character variety, ortholengths

\section{Introduction}

Let $M$ be an orientable 3-manifold which admits a complete, finite-volume hyperbolic structure with a single cusp. Thurston's hyperbolic Dehn surgery theorem applied to $M$ says that all but a finite number of topological Dehn fillings on $M$ have hyperbolic structures. To prove this, Thurston introduced the deformation space $\mathcal{H}(M)$ of incomplete hyperbolic structures on $M$ whose completions have 'Dehn surgery-type' singularities (see [25]). This space includes any hyperbolic cone-manifold whose set of non-singular points is diffeomorphic to $M$. The space $\mathcal{H}(M)$ is of continuing interest because it offers a possible approach (due to Thurston) to the Geometrization Conjecture. In particular, non-hyperbolic geometric structures and topological decompositions along incompressible spheres and tori can often be produced by understanding the ways that hyperbolic structures can degenerate near the boundary of $\mathcal{H}(M)$ (see Thurston [25, Chapter 4], Kerckhoff [18] and Kojima [20]). This approach has recently yielded a proof of the Orbifold Theorem (see Cooper-HodgsonKerckhoff [6, Chapter 7] or Boileau-Porti [2]). 
In this paper we introduce a $\mathbb{C}^{n}$-valued invariant Orth $_{K}$ of each incomplete hyperbolic structure in $\mathcal{H}(M)$, called the ortholength invariant. This invariant is defined in terms of a topological ideal triangulation $K$ of $M$, but it is actually independent of $K$ in the sense that for 'generic' $K$, Orth $K$ determines the ortholength invariant corresponding to any other ideal triangulation (see Theorem 17). The main result of this paper says that away from a (possibly empty) subvariety of values, the ortholength invariant locally parameterises $\mathcal{H}(M)$ and it is a complete invariant of the (topological) Dehn fillings of $M$ which admit a hyperbolic structure (see Theorem 18).

For a hyperbolic structure whose metric completion is a cone-manifold, the ortholength invariant is essentially a list of hyperbolic cosines of the complex distances $^{1}$ from the cone-manifold's singular set to itself along the edges ${ }^{2}$ of $K$. Hence there is a close connection between the ortholength invariant and the tube radius of a hyperbolic Dehn filling, i.e. the supremum of the radii of the embedded hyperbolic tubes in $M$ about the singular set of the Dehn filling.

The importance of the tube radius has emerged recently from the work of Kojima [19] and Hodgson-Kerckhoff [18], [17]. In these papers, the condition that the tube radius stays bounded away from zero as cone-angles are varied has emerged as the key to ensuring that the hyperbolic structures do not degenerate. (By contrast, it is possible for hyperbolic cone-manifolds to degenerate while their volume remains bounded above zero, e.g. see [19], especially Theorem 7.1.2 or the example of $\S 7.2$.) This suggests a relationship between the boundary of $\mathcal{H}(M)$ and the ortholength invariant which deserves further study (see Conjecture 20).

The terminology 'ortholength invariant' follows [21], where Meyerhoff defines the complex ortholength spectrum of a closed hyperbolic 3-manifold as the set of complex distances between pairs of the manifold's simple closed geodesics. He shows that the ortholength spectrum plus some combinatorial data determines the manifold up to isometry. Hence the fact that $\operatorname{Orth}_{K}$ is a complete invariant of the topological Dehn fillings of $M$ which admit a hyperbolic structure can be interpreted as a strengthened version of Meyerhoff's result, namely that a finite (and computable) subset of the ortholength spectrum plus slightly stronger combinatorial information than assumed in [21] determines the filled manifold up to isometry.

\footnotetext{
${ }^{1}$ The complex distance between two lines is the hyperbolic distance between them plus $i$ times an angle of twist.

${ }^{2}$ Not all homotopy classes of paths from the singular set to itself contain a distancerealising geodesic, however the ortholength invariant is defined purely in terms of the holonomy representation and so is well-defined in any case.
} 
The rest of this paper is set out as follows. In Section 2 we show that it is possible to parameterise a configuration of lines in $\mathbb{H}^{3}$ in terms of the complex distances between them. In Section 3 we define the ortholength invariant and give a formula for it in terms of the traces of the holonomies of certain loops in $M$, thereby showing that Orth $_{K}$ is a rational map from the $\mathrm{PSL}_{2} \mathbb{C}$-character variety $X(M)$ of $M$ into $\mathbb{C}^{n}$. The main section of this paper is Section 4, where we prove that the ortholength invariant locally parameterises incomplete hyperbolic structures on $M$ and is a complete invariant when restricted to the (topological) Dehn fillings of $M$ which admit a hyperbolic structure (for those hyperbolic structures whose ortholength invariant does not lie in a certain subvariety). We also prove (under a weak technical assumption) that the ortholength invariant is a birational equivalence from certain interesting irreducible components of $X(M)$ to certain irreducible components of a complex affine algebraic variety $\mathcal{P}(K) \subseteq \mathbb{C}^{n}$. In Section 5 we calculate the ortholength invariant when $M$ is the figure- 8 knot complement. Section 6 concludes this paper with a conjecture and a discussion of some applications for the ortholength invariant, including the construction of incomplete hyperbolic structures on $M$.

This paper is based on my doctoral thesis and I would like to acknowledge and thank my advisor Craig D. Hodgson for his guidance throughout the work presented here.

\section{Configurations of lines in hyperbolic 3-space}

We say that $n$ oriented lines $l_{1}, \ldots, l_{n}$ in hyperbolic 3 -space $\mathbb{H}^{3}$ realise a set of complex numbers $x_{i j} \in \mathbb{C}(i, j=1, \ldots, n)$ if cosh of the complex distance ${ }^{3}$ between each pair of lines $l_{i}$ and $l_{j}$ is equal to $x_{i j}$. This section is motivated by the following question.

Problem 1 When is it possible to realise a given set of complex numbers $x_{i j} \in \mathbb{C}(i, j=1, \ldots, n)$ by an arrangement of lines and how unique is such an arrangement?

A complete answer to Problem 1 is given in Theorem 7. A corollary to this theorem (Corollary 8) says that there exists an arrangement of four lines which realises a set of complex numbers $x_{i j} \in \mathbb{C}(i, j=1, \ldots, 4)$ if and only if these complex numbers satisfy a 'hextet' equation. This corollary is the key result

\footnotetext{
${ }^{3}$ The complex distance between two lines is the hyperbolic distance between them plus $i$ times an angle of twist-see Fenchel [10, $§ \mathrm{~V} .3]$.
} 
which allows us to locally parameterise hyperbolic structures on a 3-manifold by 'ortholengths' (see Section 4). The results of this section prior to Problem 4 are simply re-statements of some of the ideas Fenchel presented in [10]. For related material see Thurston $[27, \S \S 2.3-2.6]$.

The end-points of a line in $\mathbb{H}^{3}$ are two distinct points on the sphere at infinity $\mathbb{S}_{\infty}^{2}$, and conversely any two such points determine a line. We identify the set of oriented lines in $\mathbb{H}^{3}$ with $\mathbb{S}_{\infty}^{2} \times \mathbb{S}_{\infty}^{2} \backslash \Delta$ where $\Delta=\left\{(x, x) \mid x \in \mathbb{S}_{\infty}^{2}\right\}$, and we refer to an element of $\mathbb{S}_{\infty}^{2} \times \mathbb{S}_{\infty}^{2} \backslash \Delta$ as the ordered end-points of an oriented line in $\mathbb{H}^{3}$.

A simple calculation shows that every traceless matrix $A \in \mathrm{SL}_{2} \mathbb{C}$ has eigenvalues $\pm i$. The eigenvectors of $A$ correspond to points on the sphere at infinity $\mathbb{S}_{\infty}^{2}$ which are fixed by the action ${ }^{4}$ of $A$. Hence there is an oriented line corresponding to $A$ whose ordered end-points are $(p, q) \in \mathbb{S}_{\infty}^{2} \times \mathbb{S}_{\infty}^{2} \backslash \Delta$, where $p$ corresponds to eigenvalue $-i$ and $q$ corresponds to eigenvalue $i$. It is not hard to check that the map $A \mapsto(p, q)$ is a homeomorphism from

$$
\mathrm{S} \mathcal{L} \stackrel{\text { def }}{=}\left\{l \in \mathrm{SL}_{2} \mathbb{C} \mid \operatorname{tr} l=0\right\}
$$

to the space of oriented lines in $\mathbb{H}^{3}$. We identify these two spaces and from now on we will use the same symbol to denote both an oriented line and the corresponding element of $\mathrm{S} \mathcal{L}$.

Note that as a consequence of this, $-l \in \mathrm{S} \mathcal{L}$ denotes the same line in $\mathbb{H}^{3}$ as $l \in \mathrm{S} \mathcal{L}$ but with the opposite orientation.

Now, $\mathrm{PSL}_{2} \mathbb{C}$ acts by orientation-preserving isometries on the set of oriented lines in $\mathbb{H}^{3}$. Under the correspondence between oriented lines and $\mathrm{S} \mathcal{L}$, this gives us a corresponding action of $\mathrm{PSL}_{2} \mathbb{C}$ on $\mathrm{S} \mathcal{L}$, given by $g \cdot l=\tilde{g} l \tilde{g}^{-1}$ for any $g \in \mathrm{PSL}_{2} \mathbb{C}$ and $l \in \mathrm{S} \mathcal{L}$, where $\tilde{g} \in \mathrm{SL}_{2} \mathbb{C}$ is either of the matrices covering $g$. Hence studying the geometric properties of arrangements of lines in $\mathbb{H}^{3}$ is the same as studying the properties of subsets of $\mathrm{S} \mathcal{L}$ which are invariant under the conjugacy action of $\mathrm{SL}_{2} \mathbb{C}$.

Given two oriented lines $l, m \in \mathrm{S} \mathcal{L}$, an obvious conjugacy invariant of the pair is $\operatorname{tr}(l m)$. Lemma 3 (below) says that this invariant is essentially cosh of the complex distance between $l$ and $m$. However, before stating this lemma we introduce some notation ${ }^{5}$.

\footnotetext{
${ }^{4} A$ acts as a projective transformation on $\mathbb{S}_{\infty}^{2}=\mathbb{C P}^{1}$.

${ }^{5} \mathrm{I}$ am grateful to the referee for drawing my attention to the fact that $\mathcal{L}$ is the Lie algebra of $\mathrm{SL}_{2} \mathbb{C}$, the corresponding action of $\mathrm{SL}_{2} \mathbb{C}$ on $\mathcal{L}$ is the adjoint action, the form $\langle\cdot, \cdot\rangle$ (below) is a multiple of the Killing form and Lemma 5 (below) is essentially the (known) result that $\mathrm{PSL}_{2} \mathbb{C}$ is isomorphic to $\mathrm{SO}_{3} \mathbb{C}$.
} 
Definition 2 Line Matrices The complex vector space $\mathcal{L}$ of line matrices consists of all $2 \times 2$ complex matrices with zero trace. This space is endowed with a symmetric bilinear form $\langle\cdot, \cdot\rangle$ given by

$$
<l, m>=-\frac{1}{2} \operatorname{tr}(l m)
$$

for any $l, m \in \mathcal{L}$.

It is not hard to check that the bilinear form $\langle\cdot, \cdot\rangle$ is non-degenerate on $\mathcal{L}$. Also, each non-singular line matrix $l \in \mathcal{L}$ acts on $\mathbb{H}^{3}$ as a half-turn about some line so there is an (unoriented) line associated with each non-singular line matrix. This is where the terminology 'line matrices' comes from.

Lemma 3 For any $l, m \in S \mathcal{L}$,

$$
<l, m>=\cosh \left(d_{\mathbb{C}}(l, m)\right)
$$

where $d_{\mathbb{C}}(l, m)$ is the complex distance between $l$ and $m$.

Proof See Fenchel [10, §V.3].

Alternatively, the reader can take Lemma 3 as the definition of the complex distance between two oriented lines in $\mathbb{H}^{3}$.

Note that $\langle l, l\rangle=\operatorname{det} l$ for any $l \in \mathcal{L}$ so $\mathrm{S} \mathcal{L}=\{l \in \mathcal{L} \mid\langle l, l\rangle=1\}$ is the set of normalised line matrices. Then by Lemma 3 we can rephrase Problem 1 in terms of linear algebra as follows.

Problem 4 Given some complex numbers $x_{i j} \in \mathbb{C}(i, j=1, \ldots, n)$ so that $x_{i j}=x_{j i}$ and $x_{i i}=1$, do there exist oriented lines $l_{1}, \ldots, l_{n} \in \mathrm{S} \mathcal{L}$ so that

$$
<l_{i}, l_{j}>=x_{i j} ?
$$

To what extent do these conditions determine the lines $l_{1}, \ldots, l_{n}$ if they exist?

Now, the action of $\mathrm{PSL}_{2} \mathbb{C}$ on $\mathrm{S} \mathcal{L}$ extends to an action on $\mathcal{L}$ in an obvious way ${ }^{6}$ and clearly $\langle\cdot, \cdot\rangle$ is invariant under this action. Also note that $\langle\cdot, \cdot\rangle$ is invariant under the map $\mathcal{L} \rightarrow \mathcal{L}$ given by $l \mapsto-l$. The following lemma says that these are the only isomorphisms of $(\mathcal{L},<\cdot, \cdot>)$.

\footnotetext{
${ }^{6}$ For $g \in \mathrm{PSL}_{2} \mathbb{C}$ and $l \in \mathcal{L}, g \cdot l=\tilde{g} l \tilde{g}^{-1}$ where $\tilde{g} \in \mathrm{SL}_{2} \mathbb{C}$ is one of the two matrices covering $g$.
}

Algebraic 83 Geometric Topology, Volume 2 (2002) 
Lemma 5 For each linear map $\phi: \mathcal{L} \rightarrow \mathcal{L}$ which preserves $\langle\cdot, \cdot\rangle$ there is some element $g \in P S L_{2} \mathbb{C}$ and some choice \pm 1 of sign so that

$$
\pm \phi(l)=g \cdot l
$$

for any $l \in \mathcal{L}$.

Proof Consider three lines $l_{1}, l_{2}, l_{3} \in \mathrm{S} \mathcal{L}$ for which $\left\langle l_{i}, l_{j}\right\rangle=0$ for each $i \neq j$. By Lemma 3, these lines all meet at a common point of $\mathbb{H}^{3}$ where they are mutually perpendicular. We assume that the orientations of the $l_{i}$ have been chosen so that they define a right-handed frame at this common point (see Figure 1).

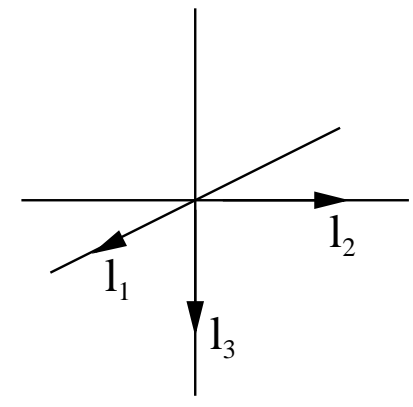

(a) Left-handed

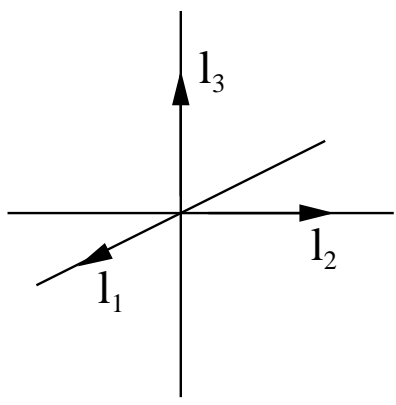

(b) Right-handed

Figure 1: The two orientation-classes of orthogonal frames

Now, if $\phi: \mathcal{L} \rightarrow \mathcal{L}$ preserves $\left\langle\cdot, \cdot>\right.$ then $\left\langle\phi\left(l_{i}\right), \phi\left(l_{j}\right)>=0\right.$ for each $i \neq j$ and so by Lemma $3, \phi\left(l_{1}\right), \phi\left(l_{2}\right), \phi\left(l_{3}\right)$ are also mutually perpendicular lines which meet at a common point. By replacing $\phi$ by $-\phi$ if need be we can assume $\phi\left(l_{1}\right), \phi\left(l_{2}\right), \phi\left(l_{3}\right)$ define a right-handed frame. Since $\mathrm{PSL}_{2} \mathbb{C}$ acts transitively on the bundle of right-handed orthogonal frames of $\mathbb{H}^{3}$ (see $[27, \S 2.2]$ ) there is an isometry $g \in \mathrm{PSL}_{2} \mathbb{C}$ which takes $l_{i}$ to $\phi\left(l_{i}\right)$ for each $i=1,2,3$. Hence $\phi\left(l_{i}\right)=$ $g \cdot l_{i}$ for each $i=1,2,3$. Since the $l_{i}$ form a basis for $\mathcal{L}$ (linear dependence would imply that $\left.<l_{i}, l_{i}\right\rangle=0$ for some $i$ ) this proves the lemma.

In fact, from this proof it is clear that the action of $\mathrm{PSL}_{2} \mathbb{C} \times\{ \pm 1\}$ on $\mathcal{L}$ induces an isomorphism from $\mathrm{PSL}_{2} \mathbb{C} \times\{ \pm 1\}$ to the group $\mathrm{O}_{3}(\mathbb{C})$ of isomorphisms of $(\mathcal{L},<\cdot, \cdot>)$ (see also Footnote 5 , above). Note that -1 acts on $\mathcal{L}$ by simultaneously reversing the orientations of all lines and is not related to the orientation-reversing isometries of $\mathbb{H}^{3}$. 
Let $(V, g)$ denote a finite-dimensional complex vector space $V$ equipped with a symmetric, bilinear form $g$. Then associated to $g$ there is a map $V \rightarrow V^{*}$ from $V$ to its dual given by $v \mapsto g(v, \cdot)$. The rank of $g$ (denoted $\operatorname{rank}(g)$ ) is the dimension of the image of this map and $g$ is non-degenerate if this map is an isomorphism. To solve Problem 4 we will use the following lemma from linear algebra.

Lemma 6 Let $(V, g)$ and $(W, h)$ be two finite-dimensional complex vector spaces equipped with symmetric, bilinear forms $g$ and $h$. If $\operatorname{rank}(g) \leq \operatorname{rank}(h)$ then there exists a linear transformation $\phi: V \rightarrow W$ so that

$$
\phi^{*} h=g
$$

i.e. so that $g(x, y)=h(\phi x, \phi y)$ for any $x, y \in V$. Furthermore, if $h$ is nondegenerate and $\operatorname{rank}(g)=\operatorname{rank}(h)$ or $\operatorname{rank}(g)=\operatorname{rank}(h)-1$ then $\phi$ is unique up to composing it on the left with an isomorphism of $(W, h)$.

Proof This is an elementary consequence of the fact that $(V, g)$ is isomorphic to $\left(\mathbb{C}^{n}, E_{r}\right)$ for $n=\operatorname{dim}(V)$ and $r=\operatorname{rank}(g)$, where $E_{r}$ is the bilinear form defined by $E_{r}(u, v)=u^{\mathrm{T}} G v$ for any $u, v \in \mathbb{C}^{n}$ and $G$ is the $n \times n$ diagonal matrix $\operatorname{diag}(1, \ldots, 1,0, \ldots, 0)$ with $r$ non-zero entries.

Armed with Lemmas 5 and 6 we can now completely solve Problem 4.

Theorem 7 For $i, j=1, \ldots, n$ let $x_{i j} \in \mathbb{C}$ be given complex numbers so that $x_{i j}=x_{j i}$ and $x_{i i}=1$. Then there exist $l_{1}, \ldots, l_{n} \in S \mathcal{L}$ for which $\left\langle l_{i}, l_{j}\right\rangle=x_{i j}$ if and only if $\operatorname{rank}(X) \leq 3$, where $X$ is the $n \times n$ matrix whose $(i, j)^{\text {th }}$ entry is $x_{i j}$. If some $x_{i j} \neq \pm 1$ then the arrangement of lines $l_{1}, \ldots, l_{n}$ is unique up to the action of $P S L_{2} \mathbb{C} \times\{ \pm 1\}$, i.e. unique up to orientation-preserving isometry and simultaneous reversal of orientations.

If all $x_{i j}= \pm 1$ then there may be non-isometric arrangements of lines which realise the $x_{i j}$.

Proof of Theorem 7 Let $x_{i j} \in \mathbb{C}$ be given complex numbers and suppose that there exist lines $l_{1}, \ldots, l_{n} \in \mathrm{S} \mathcal{L}$ so that $x_{i j}=<l_{i}, l_{j}>$. Then since $\mathcal{L}$ is 3 -dimensional, any four of the lines must be linearly dependent. From this it follows that any four rows of $X$ are linearly dependent. For example, there exist non-zero constants $\alpha_{1}, \ldots, \alpha_{4} \in \mathbb{C}$ so that $\alpha_{1} l_{1}+\ldots+\alpha_{4} l_{4}=0$ and hence

$$
\alpha_{1}<l_{1}, l_{i}>+\ldots+\alpha_{4}<l_{4}, l_{i}>=0
$$


(i.e. $\alpha_{1} x_{1 i}+\ldots+\alpha_{4} x_{4 i}=0$ ) for each $i=1, \ldots, n$. Hence $\operatorname{rank}(X) \leq 3$.

So now suppose that for $i, j=1, \ldots, n$ we have $x_{i j} \in \mathbb{C}$ so that $x_{i j}=x_{j i}$ and $x_{i i}=1$ and that $\operatorname{rank}(X) \leq 3$ where $X=\left[x_{i j}\right]$ is the matrix defined in the statement. Define a symmetric, bilinear form $g$ on $\mathbb{C}^{n}$ by

$$
g(x, y)=x^{\mathrm{T}} X y
$$

for any $x, y \in \mathbb{C}^{n}$. Then $\operatorname{rank}(g) \leq 3=\operatorname{rank}(<\cdot, \cdot>)$ so setting $(V, g)=\left(\mathbb{C}^{n}, g\right)$ and $(W, h)=(\mathcal{L},<\cdot, \cdot>)$ in Lemma 6 gives us a linear map $\phi: \mathbb{C}^{n} \rightarrow \mathcal{L}$ so that $\phi^{*}<\cdot, \cdot>=g$. For each $i=1, \ldots, n$ let $l_{i} \stackrel{\text { def }}{=} \phi e_{i}$ where $e_{1}, \ldots, e_{n}$ is the standard basis for $\mathbb{C}^{n}$. Then

$$
x_{i j}=e_{i}{ }^{\mathrm{T}} X e_{j}=g\left(e_{i}, e_{j}\right)=<\phi e_{i}, \phi e_{j}>=<l_{i}, l_{j}>
$$

for any $i, j=1, \ldots, n$. Note that $\left\langle l_{i}, l_{i}\right\rangle=x_{i i}=1$ for each $i=1, \ldots n$ so $l_{i} \in \mathrm{S} \mathcal{L}$, i.e. each $l_{i}$ corresponds to an oriented line in $\mathbb{H}^{3}$.

Now, if some $x_{i j} \neq \pm 1$ then $\operatorname{rank}(g)=2$ or 3 (this uses the fact that $X$ is symmetric and $x_{i i}=1$ ). Hence by Lemma $6, \phi$ (and hence the arrangement of lines $\left.l_{1}, \ldots, l_{n}\right)$ is unique up to composing $\phi$ by an isomorphism of $(\mathcal{L},<\cdot, \cdot>)$. But by Lemma 5 the isomorphisms of $(\mathcal{L},<\cdot, \cdot>)$ are exactly given by the action of $\mathrm{PSL}_{2} \mathbb{C} \times\{ \pm 1\}$ on $\mathcal{L}$. Hence the arrangement of lines $l_{1}, \ldots, l_{n}$ is unique up to orientation-preserving isometry and simultaneous reversal of orientations.

The special cases of Theorem 7 when $n=3$ and $n=4$ are of particular interest to us. If three lines $l_{1}, l_{2}, l_{3} \in \mathrm{S} \mathcal{L}$ all have distinct end-points on $\mathbb{S}_{\infty}^{2}$ then any two of these lines has a common-perpendicular. Adding these three perpendiculars and truncating appropriately gives an arrangement of six line segments in $\mathbb{H}^{3}$ which meet at right-angles, i.e. a right-angled hexagon (see Figure 2(a)). Motivated by this generic case we will simply say that any three oriented lines define a right-angled hexagon, without making the assumption that the lines have distinct end-points. Then Theorem 7 applied to $l_{1}, l_{2}, l_{3}$ gives the well-known result that the complex distances along alternating edges of a right-angled hexagon determine the hexagon up to isometry (see [10, §VI.4]).

Similarly, any four lines $l_{1}, \ldots, l_{4} \in \mathrm{S} \mathcal{L}$ which all have distinct end-points on $\mathbb{S}_{\infty}^{2}$ have six pair-wise common-perpendiculars. Adding these perpendiculars and truncating all lines appropriately gives an arrangement of lines in $\mathbb{H}^{3}$ loosely resembling a tetrahedron (see Figure $2(\mathrm{~b})$ ). Since this arrangement is like a tetrahedron whose vertices have been stretched into the lines $l_{1}, \ldots, l_{4}$, turning its faces into right-angled hexagons, we call such an arrangement a hextet. As with right-angled hexagons, we will drop the requirement that the end-points of $l_{1}, \ldots, l_{4}$ be distinct and simply say that any four oriented lines in $\mathbb{H}^{3}$ define a hextet. Substituting $n=4$ into Theorem 7 gives the following corollary. 


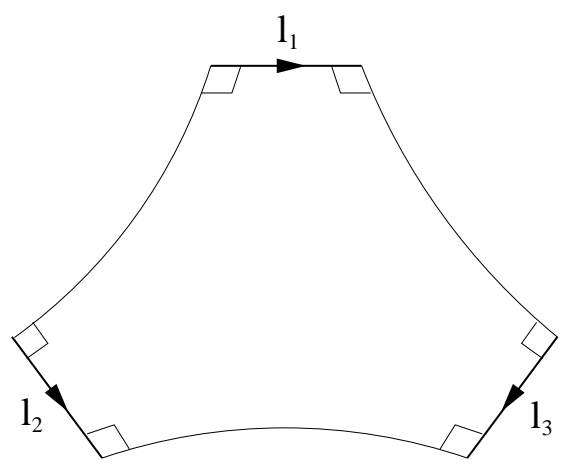

(a) A right-angled hexagon

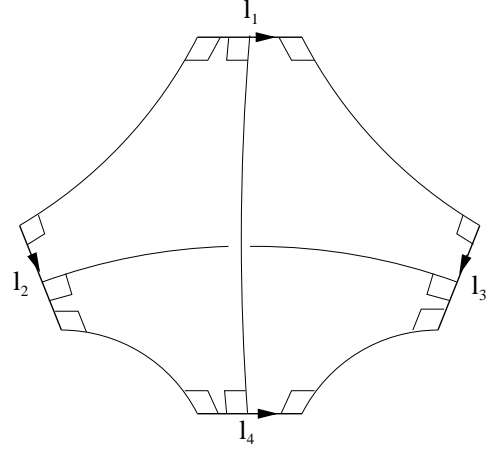

(b) A hextet

Figure 2: Arrangements of (a) three lines and (b) four lines

Corollary 8 (Existence and Rigidity of Hextets) Let $x_{i j} \in \mathbb{C}$ be given complex numbers for $i, j=1, \ldots, 4$ so that $x_{i j}=x_{j i}, x_{i i}=1$. Then there exist $l_{1}, \ldots, l_{4} \in S \mathcal{L}$ so that $\left\langle l_{i}, l_{j}\right\rangle=x_{i j}$ if and only if the $x_{i j}$ satisfy the hextet equation

$$
0=\operatorname{det}\left[\begin{array}{cccc}
1 & x_{12} & x_{13} & x_{14} \\
x_{21} & 1 & x_{23} & x_{24} \\
x_{31} & x_{32} & 1 & x_{34} \\
x_{41} & x_{42} & x_{43} & 1
\end{array}\right] .
$$

If some $x_{i j} \neq \pm 1$ then these lines $l_{1}, \ldots, l_{4}$ are unique up to orientationpreserving isometry and simultaneous reversal of each line's orientation.

It was known to Fenchel (see $[10, \S \mathrm{V} .3]$ ) that if four lines $l_{1}, \ldots, l_{4} \in \mathrm{S} \mathcal{L}$ are given then the complex numbers $<l_{i}, l_{j}>$ satisfy the above hextet equation.

We finish this section with a brief discussion of degenerate arrangements of lines in $\mathbb{H}^{3}$.

Definition 9 An arrangement of lines $l_{1}, \ldots, l_{n} \in \mathrm{S} \mathcal{L}$ is non-degenerate if $l_{1}, \ldots, l_{n}$ spans $\mathcal{L}$ and the arrangement is degenerate otherwise.

The following lemma gives us three more characterisations of degeneracy.

Lemma 10 Let $l_{1}, \ldots, l_{n} \in S \mathcal{L}$ be given, let $x_{i j}=\left\langle l_{i}, l_{j}>\right.$ for each $i, j=$ $1, \ldots, n$ and let $X$ be the $n \times n$ matrix whose $(i, j)^{t h}$ entry is $x_{i j}$. If some $x_{i j} \neq \pm 1$ then the following are equivalent: 
(1) $l_{1}, \ldots, l_{n}$ is a degenerate arrangement of lines.

(2) $l_{1}, \ldots, l_{n}$ have a common perpendicular line.

(3) There exists an orientation-preserving isometry taking the arrangement $l_{1}, \ldots, l_{n}$ to the arrangement $-l_{1}, \ldots,-l_{n}$.

(4) $\operatorname{rank}(X)=2$.

Proof $(1) \Rightarrow(2)$ Suppose that $l_{1}, \ldots, l_{n}$ are linearly dependent. Some $x_{i j} \neq$ \pm 1 so without loss of generality we assume $x_{12} \neq \pm 1$ and hence that $l_{1}$ and $l_{2}$ have no end-points in common. Therefore $l_{1}$ and $l_{2}$ are linearly independent and they also have a common perpendicular $n \in \mathrm{S} \mathcal{L}$ (whose orientation is not unique). Then for each $i=3, \ldots, n$ there exist $\alpha_{1}, \alpha_{2} \in \mathbb{C}$ so that $l_{i}=$ $\alpha_{1} l_{1}+\alpha_{2} l_{2}$. But $\left\langle l_{1}, n\right\rangle=\left\langle l_{2}, n\right\rangle=0$ and so $\left\langle l_{i}, n\right\rangle=0$ and hence $l_{i}$ is also perpendicular to $n$ (by Lemma 3 ).

(2) $\Rightarrow$ (3) If $l_{i} \in \mathrm{S} \mathcal{L}$ is perpendicular to $n \in \mathrm{S} \mathcal{L}$ then the half-turn about $n$ takes $l_{i}$ to $-l_{i}$.

(3) $\Rightarrow$ (1) Suppose there is some $g \in \mathrm{PSL}_{2} \mathbb{C}$ so that $g \cdot l_{i}=-l_{i}$ for each $i=1, \ldots, n$. If $l_{1}, \ldots, l_{n}$ span $\mathcal{L}$ then $g$ is an isometry which takes every oriented line to the same line but with the opposite orientation, which is absurd.

$(1) \Rightarrow$ (4) If we assume that any three of the lines $l_{1}, \ldots, l_{n}$ are linearly dependent then it follows that any three of the rows of $X$ are linearly dependent, too, and hence that $\operatorname{rank}(X)<3$. For example, if $\alpha_{1} l_{1}+\alpha_{2} l_{2}+\alpha_{3} l_{3}=0$ then

$$
\begin{aligned}
0 & =\alpha_{1}<l_{1}, l_{i}>+\alpha_{2}<l_{2}, l_{i}>+\alpha_{3}<l_{3}, l_{i}> \\
& =\alpha_{1} x_{1 i}+\alpha_{2} x_{2 i}+\alpha_{3} x_{3 i}
\end{aligned}
$$

for each $i=1, \ldots, n$. But since some $x_{i j} \neq \pm 1, \operatorname{rank}(X) \neq 1$ and so $\operatorname{rank}(X)=2$.

(4) $\Rightarrow$ (1) Conversely, suppose that $\operatorname{rank}(X)=2$ and assume (in order to derive a contradiction) that the $l_{1}, \ldots, l_{n}$ span $\mathcal{L}$. Without loss of generality assume that $l_{1}, l_{2}, l_{3}$ form a basis for $\mathcal{L}$. Then since $\operatorname{rank}(X)=2$, the first three rows of $X$ are linearly dependent so there exist $\alpha_{1}, \alpha_{2}, \alpha_{3} \in \mathbb{C}$ so that

$$
\begin{aligned}
0 & =\alpha_{1} x_{1 i}+\alpha_{2} x_{2 i}+\alpha_{3} x_{3 i} \\
& =<\alpha_{1} l_{1}+\alpha_{2} l_{2}+\alpha_{3} l_{3}, l_{i}>
\end{aligned}
$$

for each $i=1, \ldots, n$. But since $<\cdot, \cdot\rangle$ is non-degenerate and (by assumption) $l_{1}, \ldots, l_{n} \operatorname{span} \mathcal{L}$, this implies $\alpha_{1} l_{1}+\alpha_{2} l_{2}+\alpha_{3} l_{3}=0$ which is a contradiction. 


\section{The ortholength invariant}

In this section we define the ortholength invariant of each incomplete hyperbolic structure in the hyperbolic Dehn surgery space $\mathcal{H}(M)$ of $M$. This invariant is given purely in terms of holonomy representations so its definition naturally extends to a map Orth $_{K}: X(M) \rightarrow \mathbb{C}^{n}$ from the $\mathrm{PSL}_{2} \mathbb{C}$-character variety $X(M)$ of $M$ to $\mathbb{C}^{n}$. We show that Orth $_{K}$ is a rational map whose image lies inside a variety $\mathcal{P}(K) \subseteq \mathbb{C}^{n}$. For examples of $\operatorname{Orth}_{K}, \mathcal{P}(K)$ and $X(M)$, see Section 5 .

Let $M$ be an oriented, finite volume, 1-cusped hyperbolic 3-manifold (as in Section 1) and let $N$ be an embedded horoball neighbourhood of the cusp. Then $N$ is diffeomorphic to the product of a 2-torus $T^{2}$ with the half-open interval $[0,1)$ and furthermore the complement in $M$ of the interior of $N$ is a compact 3 -manifold with boundary $\partial N \cong T^{2}$ (e.g. see Thurston [27, §4.5]). Let $K$ be a (topological) ideal triangulation of $M$ (see Benedetti-Petronio [1, $\S$ E.5-i]) which meets $N$ 'nicely', i.e. so that inside any tetrahedron $\triangle$ of $K, N$ has four connected components, each being a punctured neighbourhood of one of the vertices of $\triangle$. Let $*$ be a base-point for $M$ which lies in $\partial N$.

Now, let $\rho: \pi_{1}(M, *) \rightarrow \mathrm{PSL}_{2} \mathbb{C}$ be a homomorphism which satisfies the condition that $\rho\left(\pi_{1}(\partial N, *)\right)$ fixes exactly two points on the sphere at infinity $\mathbb{S}_{\infty}^{2}$ (e.g. $\rho$ could be the holonomy representation of an incomplete hyperbolic structure of $\mathcal{H}(M))$. A simple investigation of the fixed-points of the Abelian group $\rho\left(\pi_{1}(\partial N, *)\right)$ shows that this condition is equivalent to the requirement that $\rho\left(\pi_{1}(\partial N, *)\right)$ is a non-trivial group of non-parabolic ${ }^{7}$ isometries which is not isomorphic to $\mathbb{Z} / 2 \oplus \mathbb{Z} / 2$ (where $\mathbb{Z} / 2$ is the group with two elements). These conditions in turn are equivalent to the algebraic conditions that

$$
\begin{array}{r}
\text { neither } \operatorname{tr}^{2} \rho(m)=\operatorname{tr}^{2} \rho(l)=4 \\
\text { nor } \operatorname{tr}^{2} \rho(m)=\operatorname{tr}^{2} \rho(l)=\operatorname{tr}^{2} \rho(m l)=0,
\end{array}
$$

where $m$ and $l$ are any pair of generators of $\pi_{1}(\partial N, *) \cong \mathbb{Z} \oplus \mathbb{Z}$.

Now, by our assumption that $\rho$ satisfies the conditions (2), it follows that the group $\rho\left(\pi_{1}(\partial N, *)\right)$ fixes a unique line in $\mathbb{H}^{3}$. Choose an orientation and call the resulting oriented line $\sigma$. Let $\pi: \widetilde{M} \rightarrow M$ be the universal cover of $M$ and choose some base-point $\tilde{*} \in \pi^{-1}(*)$. This choice allows us to identify the deck-transformations of $\pi: \widetilde{M} \rightarrow M$ with $\pi_{1}(M, *)$. Let $\widetilde{N}$ be the connected component of $\pi^{-1}(N)$ which contains $\tilde{*}$.

\footnotetext{
${ }^{7}$ An orientation-preserving isometry $g \in \mathrm{PSL}_{2} \mathbb{C}$ is parabolic if $g \neq 1$ and $\operatorname{tr}^{2} g=4$.
} 
Let $e_{1}, \ldots, e_{n}$ be the edges of $K$ and for each $i=1, \ldots, n$ choose a lift $\tilde{e}_{i}$ of edge $e_{i}$ in $\widetilde{M}$. To each end of $\tilde{e}_{i}$ there is a corresponding connected component of $\pi^{-1}(N)$. Denote these connected components by $\widetilde{N}_{1}$ and $\widetilde{N}_{2}$ (note that it is possible that $\widetilde{N}_{1}=\widetilde{N}_{2}$ if $e_{i}$ is homotopically trivial). Then since $\widetilde{N}_{1}$ and $\widetilde{N}_{2}$ both cover $N$, there exist deck-transformations $\gamma_{1}$ and $\gamma_{2}$ for which $\gamma_{i}(\widetilde{N})=\widetilde{N}_{i}$ (for each $i=1,2)$. We define the ortholength $d_{i}$ corresponding to edge $e_{i}$ to be

$$
d_{i} \stackrel{\text { def }}{=} d_{\mathbb{C}}\left(\rho\left(\gamma_{1}\right) \cdot \sigma, \rho\left(\gamma_{2}\right) \cdot \sigma\right)
$$

i.e. define $d_{i}$ to be the complex distance between $\rho\left(\gamma_{1}\right) \cdot \sigma$ and $\rho\left(\gamma_{2}\right) \cdot \sigma$. While $\gamma_{1}$ and $\gamma_{2}$ are not unique, $\rho\left(\gamma_{1}\right) \cdot \sigma$ and $\rho\left(\gamma_{2}\right) \cdot \sigma$ do not depend on their arbitrariness and so are well-defined oriented lines in $\mathbb{H}^{3}$. Also, the definition of $d_{i}$ doesn't depend on our choice of lift $\tilde{e}_{i}$ of edge $e_{i}$. This is because any other lift is of the form $\alpha \cdot \tilde{e}_{i}$ for some deck-transformation $\alpha$. In the above prescription this has the effect of replacing $\widetilde{N}_{i}$ by $\alpha\left(\widetilde{N}_{i}\right)$, i.e. replacing $\gamma_{i}$ by $\alpha \gamma_{i}(i=1,2)$. But since $d_{\mathbb{C}}\left(\rho(\alpha) \rho\left(\gamma_{1}\right) \cdot \sigma, \rho(\alpha) \rho\left(\gamma_{2}\right) \cdot \sigma\right)=d_{\mathbb{C}}\left(\rho\left(\gamma_{1}\right) \cdot \sigma, \rho\left(\gamma_{2}\right) \cdot \sigma\right)$, $d_{i}$ is not affected by this change.

Also, conjugating $\rho$ by some $g \in \mathrm{PSL}_{2} \mathbb{C}$ has the effect of replacing $\sigma$ by $g \cdot \sigma$ and each $\rho\left(\gamma_{1}\right)$ by $g \rho\left(\gamma_{1}\right) g^{-1}$, which clearly leaves $d_{i}$ unchanged. This shows that $d_{i}$ is independent of our choice of $\tilde{*}$ (which we used to identify $\pi_{1}(M, *$ ) with the deck-transformations of $\widetilde{M} \rightarrow M$ ) and also that $d_{i}$ only depends on the conjugacy class of $\rho$. We define the ortholength invariant $\operatorname{Orth}_{K}(\rho)$ with respect to the ideal triangulation $K$ evaluated at $\rho$ to be

$$
\operatorname{Orth}_{K}(\rho) \stackrel{\text { def }}{=}\left(\cosh d_{1}, \ldots, \cosh d_{n}\right) \in \mathbb{C}^{n}
$$

We think of $\operatorname{Orth}_{K}(\cdot)$ as the function which associates cosh of the ortholength $d_{i}$ to edge $e_{i}$ of $K$ for each $i=1, \ldots, n$.

Now, as noted in Footnote 5 (and see also [3]) $\mathrm{PSL}_{2} \mathbb{C}$ is naturally isomorphic to $\mathrm{SO}_{3} \mathbb{C}$ and so the space of representations $\mathcal{R}(M)$ of $\pi_{1}(M, *)$ into $\mathrm{PSL}_{2} \mathbb{C}$ is a complex algebraic variety. The $\mathrm{PSL}_{2} \mathbb{C}$-character variety $X(M)$ of $M$ is the 'quotient' (in the sense of algebraic geometry, see [3, $\S \S 3,4]$ ) of $\mathcal{R}(M)$ by the conjugacy action of $\mathrm{PSL}_{2} \mathbb{C}$. This space $X(M)$ has a natural algebraic structure which makes it an affine algebraic variety whose co-ordinate ring is the ring of regular functions on $\mathcal{R}(M)$ which are invariant under the $\mathrm{PSL}_{2} \mathbb{C}$-conjugacy action.

As noted above, $\operatorname{Orth}_{K}(\cdot)$ is defined on all representations satisfying the conditions (2) and $\operatorname{Orth}_{K}(\cdot)$ is constant on conjugacy classes. Hence $\operatorname{Orth}_{K}(\cdot)$ 
descends $^{8}$ to a function defined on the complement of a proper ${ }^{9}$ sub-variety of $X(M)$. We denote this function by $\operatorname{Orth}_{K}$ as well, and we write $\operatorname{Orth}_{K}$ : $X(M) \rightarrow \mathbb{C}^{n}$ to indicate that Orth $_{K}$ is not necessarily defined ${ }^{10}$ as a settheoretic function at all points of $X(M)$.

Now, given a tetrahedron $\triangle$ of $K$, choose some tetrahedron $\widetilde{\triangle}$ in $\widetilde{M}$ which covers it. Each corner of $\widetilde{\triangle}$ meets a unique connected component of $\pi^{-1}(N)$ and these connected components determine four (not necessarily distinct) oriented lines $\rho\left(\gamma_{1}\right) \cdot \sigma, \ldots, \rho\left(\gamma_{4}\right) \cdot \sigma$ in $\mathbb{H}^{3}$, as above. The complex distance between any pair of these lines is equal to the ortholength corresponding to one of the edges of $\triangle$. Hence the four oriented lines $\rho\left(\gamma_{1}\right) \cdot \sigma, \ldots, \rho\left(\gamma_{4}\right) \cdot \sigma$ determine a hextet which realises the complex distances associated to the edges of $\triangle$. By Corollary 8 this implies that the hyperbolic cosines of these ortholengths satisfy a certain algebraic equation for each tetrahedron of $K$. Hence the image of $\operatorname{Orth}_{K}$ lies in the following (not necessarily irreducible) complex algebraic variety $\mathcal{P}(K) \subseteq \mathbb{C}^{n}$.

Definition 11 The Ortholength Space $\mathcal{P}(K)$ The ortholength space $\mathcal{P}(K) \subseteq$ $\mathbb{C}^{n}$ corresponding to a (topological) ideal triangulation $K$ of $M$ with $n$ edges is the complex affine algebraic variety consisting of those points of $\mathbb{C}^{n}$ which satisfy the hextet equations of all the tetrahedra of $K$. Here the hextet equation of a tetrahedron $\triangle$ of $K$ is

$$
0=\operatorname{det}\left[\begin{array}{cccc}
1 & x_{12} & x_{13} & x_{14} \\
x_{21} & 1 & x_{23} & x_{24} \\
x_{31} & x_{32} & 1 & x_{34} \\
x_{41} & x_{42} & x_{43} & 1
\end{array}\right]
$$

where the vertices of $\triangle$ have been numbered arbitrarily from 1 to 4 and where we denote cosh of the ortholength associated to the edge of $\triangle$ between vertices $i$ and $j$ by $x_{i j}=x_{j i}$.

\footnotetext{
${ }^{8}$ Since $X(M)$ is not simply the quotient of the representation variety $\mathcal{R}(M)$ of $M$ by the conjugacy action of $\mathrm{PSL}_{2} \mathbb{C}$ this is not immediately obvious. We need two more facts: (1) $\operatorname{Orth}_{K}(\rho)=(1, \ldots, 1) \in \mathbb{C}^{n}$ whenever $\rho$ is reducible (i.e. whenever $\rho\left(\pi_{1}(M, *)\right)$ fixes a point on the sphere at infinity $\left.\mathbb{S}_{\infty}^{2}\right)$ and (2) if $\rho$ is irreducible and $\rho, \rho^{\prime} \in \mathcal{R}(M)$ project to the same point under the algebro-geometric quotient map $\mathcal{R}(M) \rightarrow X(M)$ then $\rho$ and $\rho^{\prime}$ are conjugate (see [3, p. 753]).

${ }^{9}$ The subvariety is proper since it doesn't contain (the characters of) the holonomy representations of the (incomplete) hyperbolic structures of $\mathcal{H}(M)$.

${ }^{10}$ Warning: Since $X(M)$ is not necessarily irreducible it is possible that Orth $_{K}$ is not defined at all on some components of $X(M)$.
} 
Note that the hextet equation of $\triangle$ doesn't depend on the arbitrary numbering of its vertices.

Although $\mathcal{P}(K)$ lies in an $n$-dimensional space (one dimension for each edge of $K$ ) and is defined by $n$ hextet equations (one for each tetrahedron of $K$ ) for 'generic' $K, \mathcal{P}(K)$ has an irreducible component which is a complex curve. This follows from Theorem 17 (below) and from the fact that Dehn surgery space is diffeomorphic to $\mathbb{C}$ in a neighbourhood of the complete structure (see Thurston $[25, \S 5.5])$. Conversely, it should be possible to prove that the irreducible component of $X(M)$ which contains the complete hyperbolic structure is one-dimensional over $\mathbb{C}$ via Theorem 17 and a lemma about the dimension of $\mathcal{P}(K)$.

We finish this section with an explicit formula for the ortholength invariant. This formula is given in terms of a presentation for $\pi_{1}(M, *)$ based on the ideal triangulation $K$ of $M$. The generators for this presentation consist of generators for $\pi_{1}(\partial N, *)$ plus loops $\alpha_{i} \notin \pi_{1}(\partial N, *)$ which lie in $\partial N \cup e_{i}$, where $e_{i}$ is the $i^{\text {th }}$ edge of $K(i=1, \ldots, n)$.

Proposition 12 Let $\rho: \pi_{1}(M, *) \rightarrow P S L_{2} \mathbb{C}$ be a representation on which Orth $_{K}$ is defined (i.e. for which the conditions (2) hold) and for $i=1, \ldots, n$ let $\tilde{h}, \tilde{g}_{i} \in S L_{2} \mathbb{C}$ be matrices which cover $\rho(\beta), \rho\left(\alpha_{i}\right) \in P S L_{2} \mathbb{C}$, for $\alpha_{i}$ as above and some non-trivial $\beta \in \pi_{1}(\partial N, *)$. Then the $i^{\text {th }}$ co-ordinate $\cosh d_{i}$ of $\operatorname{Orth}_{K}(\rho) \in$ $\mathcal{P}(K) \subseteq \mathbb{C}^{n}$ is

$$
\cosh d_{i}=2 \frac{\operatorname{tr}\left(\tilde{h} \tilde{g}_{i}\right) \operatorname{tr}\left(\tilde{h}^{-1} \tilde{g}_{i}\right)-\operatorname{tr}^{2} \tilde{g}_{i}}{\operatorname{tr}^{2} \tilde{h}-4}-1
$$

where $i=1, \ldots, n$.

Note that $\operatorname{tr}\left(\tilde{h} \tilde{g}_{i}\right) \operatorname{tr}\left(\tilde{h}^{-1} \tilde{g}_{i}\right), \operatorname{tr}^{2} \tilde{g}_{i}$ and $\operatorname{tr}^{2} \tilde{h}$ are independent of the choice of matrices $\tilde{h}$ and $\tilde{g}_{i}$ covering $\rho(m)$ and $\rho\left(\alpha_{i}\right)$ and so these three functions define elements of the co-ordinate ring of $X(M)$ (see [3]). Hence from the above formula it is clear that $\operatorname{Orth}_{K}: X(M) \rightarrow \mathcal{P}(K)$ is a rational map.

Proof of Proposition 12 Suppose we have the set-up as in the statement, and let $\rho\left(\pi_{1}(\partial N, *)\right)$ fix a geodesic $\sigma$ of $\mathbb{H}^{3}$, which we give an arbitrary orientation. Then $\cosh d_{i}$ is equal to $\cosh \left(d_{\mathbb{C}}\left(\sigma, g_{i} \cdot \sigma\right)\right)$, where $g_{i}=\rho\left(\alpha_{i}\right) \in \mathrm{PSL}_{2} \mathbb{C}$. Since $\cosh \left(d_{\mathbb{C}}\left(\sigma, g_{i} \cdot \sigma\right)\right)$ is invariant under conjugating $\rho$ by an orientationpreserving isometry, we can assume

$$
\tilde{h}=\left[\begin{array}{cc}
e^{x / 2} & 0 \\
0 & e^{-x / 2}
\end{array}\right] \quad \tilde{g}_{i}=\left[\begin{array}{ll}
a & b \\
c & d
\end{array}\right],
$$


(where $a d-b c=1$ ) for the purposes of calculating $\cosh \left(d_{\mathbb{C}}\left(\sigma, g_{i} \cdot \sigma\right)\right)$. We'll then express our answer in terms which are invariant under conjugacy, and then our formula will hold true for general $\tilde{h}$ and $\tilde{g}_{i}$.

The axis of $\tilde{h}$ has end-points 0 and $\infty$ on the sphere at infinity, so the line matrix of $\sigma$ is $\pm\left[\begin{array}{cc}i & 0 \\ 0 & -i\end{array}\right] \in \mathrm{SL}_{2} \mathbb{C}$. Then by Lemma 3 and the fact that the line matrix of $g_{i} \cdot \sigma$ is $\pm \tilde{g}_{i}\left[\begin{array}{cc}i & 0 \\ 0 & -i\end{array}\right] \tilde{g}_{i}^{-1}$, we have

$$
\begin{aligned}
\cosh \left(d_{\mathbb{C}}\left(\sigma, g_{i} \cdot \sigma\right)\right) & =-\frac{1}{2} \operatorname{tr}\left[\begin{array}{cc}
i & 0 \\
0 & -i
\end{array}\right]\left[\begin{array}{ll}
a & b \\
c & d
\end{array}\right]\left[\begin{array}{cc}
i & 0 \\
0 & -i
\end{array}\right]\left[\begin{array}{cc}
d & -b \\
-c & a
\end{array}\right] \\
& =a d+b c \\
& =2 a d-1 .
\end{aligned}
$$

So our task now is to express ad invariantly. But

$$
\operatorname{tr}\left(\tilde{h} \tilde{g}_{i}\right)=e^{x / 2} a+e^{-x / 2} d \quad \text { and } \quad \operatorname{tr}\left(\tilde{h}^{-1} \tilde{g}_{i}\right)=e^{-x / 2} a+e^{x / 2} d
$$

so

$$
\begin{aligned}
\operatorname{tr}\left(\tilde{h} \tilde{g}_{i}\right) \operatorname{tr}\left(\tilde{h}^{-1} \tilde{g}_{i}\right) & =a^{2}+d^{2}+a d\left(e^{x / 2}+e^{-x / 2}\right) \\
& =(a+d)^{2}-2 a d+2 a d \cosh x \\
& =\operatorname{tr}^{2} \tilde{g}_{i}+2 a d(\cosh x-1) .
\end{aligned}
$$

Combining this with

$$
\cosh x-1=2 \cosh ^{2}(x / 2)-2=\left(\operatorname{tr}^{2} \tilde{h}-4\right) / 2
$$

gives us the required formula for $\cosh d_{i}$.

\section{Parameterising hyperbolic structures with ortho- lengths}

In this section we prove that the ortholength invariant locally parameterises the deformation space $\mathcal{H}(M)$ and is a complete invariant when restricted to the (topological) Dehn fillings of $M$ which admit a hyperbolic structure (as long as the hyperbolic structure in question has ortholength invariant not lying in a certain (possibly-empty) subvariety). We also prove (under a weak technical assumption) that the ortholength invariant is a birational equivalence from certain interesting irreducible components of $X(M)$ to certain irreducible components of $\mathcal{P}(K)$.

Fix a (topological) ideal triangulation $K$ of $M$ with $n$ edges and let a set of ortholength parameters $p=\left(p_{1}, \ldots, p_{n}\right) \in \mathcal{P}(K)$ be given. Then a realisation of 
$p$ is a set of $n$ hextets (see Figure 2(b)) each of which realises the ortholength parameters associated to the edges of a tetrahedron of $K$. More precisely, a realisation is an association of an oriented line to the four corners of each tetrahedron $\triangle$ of $K$ so that cosh of the complex distance between any pair of these four lines is equal to the ortholength parameter of the edge of $\triangle$ which lies between the corresponding pair of vertices of $\triangle$.

The broad aim of this section is to construct an inverse to Orth $_{K}: X(M) \rightarrow-\rightarrow$ $\mathcal{P}(K)$. To each point $p \in \mathcal{P}(K)$, Corollary 8 guarantees the existence of a realisation of $p$ by hextets. Our strategy for building a representation $\pi_{1}(M, *) \rightarrow$ $\mathrm{PSL}_{2} \mathbb{C}$ is essentially to glue copies of these hextets together to form a type of skeletal developing map for $M$ whose rigidity then gives us a holonomy representation for free (see the proof of Lemma 14, below). However, this approach is complicated slightly by the fact that the ortholength parameters $p=\left(p_{1}, \ldots, p_{n}\right)$ don't quite determine the hextets up to isometry (see Corollary 8 ), even if we assume no $p_{i}= \pm 1$. This forces us to make Definition 13, below.

First note that each face $F$ of $K$ is contained in exactly two tetrahedra of $K$ and that these two inclusions composed with the realisation of $p$ by hextets gives two right-angled hexagons corresponding to $F$. Here we think of a rightangled hexagon corresponding to $F$ as an association of an oriented line to each of the three corners of $F$, and by an isometry between such hexagons we mean an orientation-preserving isometry which respects this association.

Definition 13 Coherence Let $p=\left(p_{1}, \ldots, p_{n}\right) \in \mathcal{P}(K)$ be such that no $p_{i}=$ \pm 1 . Then a realisation of $p$ by hextets is coherent if the pair of right-angled hexagons corresponding to each face of $K$ are isometric. If a coherent realisation of $p$ exists then we say that $p$ is coherent.

Note that any pair of right-angled hexagons corresponding to a face of $K$ have the same ortholengths and so by Theorem 7 must be either isometric or isometric after reversing the orientations of the lines in one of the hexagons. Also note (from the definition of ortholength invariant, see Section 3) that any point in the set-theoretical image of Orth $_{K}$ is coherent.

Now, since the rigidity of hextets described in Corollary 8 may fail if all of the ortholength parameters are \pm 1 , we will usually restrict our attention to $p \in \mathcal{P}(K) \backslash \mathcal{T}$, where

$$
\mathcal{T}=\left\{\left(p_{1}, \ldots, p_{n}\right) \in \mathcal{P}(K) \mid p_{i}= \pm 1 \text { for some } i=1, \ldots, n\right\} .
$$


Also, let $F_{1}, \ldots, F_{2 n}$ denote the faces of $K$ and for each $m=1, \ldots, 2 n$ define

$$
\mathcal{S}_{m} \stackrel{\text { def }}{=}\left\{p \in \mathcal{P}(K) \mid \operatorname{det}\left[\begin{array}{ccc}
1 & p_{i} & p_{j} \\
p_{i} & 1 & p_{k} \\
p_{j} & p_{k} & 1
\end{array}\right]=0\right\}
$$

where $p_{i}, p_{j}, p_{k}$ are the co-ordinates of $p=\left(p_{1}, \ldots, p_{n}\right)$ corresponding to the edges of $F_{m}$. By Lemma 10, $\mathcal{S}_{m}$ is the set of ortholengths $p \in \mathcal{P}(K)$ so that any realisation of $p$ by hextets has a degenerate hexagon corresponding to face $F_{m}$. Define

$$
\mathcal{S} \stackrel{\text { def }}{=} \bigcup_{i \neq j} \mathcal{S}_{i} \cap \mathcal{S}_{j}
$$

i.e. a point of $\mathcal{P}(K)$ lies in $\mathcal{S}$ only if it has multiple degeneracies. Note that if $\mathcal{P}(K)$ is one complex-dimensional (which Theorem 17 indicates whenever $M$ has a single cusp) then dimensional considerations suggest $\mathcal{S}$ will be empty.

The following lemma shows how the existence and rigidity of hextets (Corollary 8) allows us to construct holonomy representations from coherent ortholength parameters.

Lemma 14 To each coherent realisation of $p \in \mathcal{P}(K) \backslash \mathcal{T}$ by hextets there exists a corresponding representation $\rho: \pi_{1}(M, *) \rightarrow P S L_{2} \mathbb{C}$ so that $\operatorname{Orth}_{K}(\rho)=$ $p$. Furthermore, up to conjugacy there are at most a finite number of representations $\rho$ for which $\operatorname{Orth}_{K}(\rho)=p$ and there is only one if $p \notin \mathcal{S}$.

Hence this lemma implies that, in $\mathcal{P}(K) \backslash \mathcal{T}$, the image of $\operatorname{Orth}_{K}: X(M)-\rightarrow$ $\mathcal{P}(K)$ is exactly the set of coherent ortholength parameters.

Before proving Lemma 14 we pause briefly to describe part of the relationship between Thurston and SnapPea's parameterisation of $X(M)$ via ideal hyperbolic tetrahedra (see [25], [28], [22]) and the parameterisation of $X(M)$ described in Lemma 14 via ortholengths $p \in \mathcal{P}(K)$. To each hextet there is an associated ideal hyperbolic tetrahedron whose vertices are the second endpoints $^{11}$ of the four oriented lines comprising the hextet. So given a coherent realisation of $p \in \mathcal{P}(K)$, we can associate a hyperbolic ideal tetrahedron to each (topological) ideal tetrahedron of $K$. Then SnapPea's edge-consistency conditions are automatically satisfied for these geometric tetrahedra. To see this, consider all the tetrahedra of $K$ surrounding an edge $e_{i}$ of $K$. In a hextet which realises one of these tetrahedra, two of the four lines comprising the hextet correspond to edge $e_{i}$ (and hence cosh of the complex distance between

\footnotetext{
${ }^{11}$ The second end-point of an oriented line $(p, q) \in \mathbb{S}_{\infty}^{2} \times \mathbb{S}_{\infty}^{2} \backslash \Delta$ is $q$.
} 
these two lines is $p_{i}$ ). By acting by (orientation-preserving) isometries if need be we can assume that these two lines are the same for each hextet. But then the pair of right-angled hexagons corresponding to a face $F$ of $K$ containing edge $e_{i}$ must also coincide (since they are isometric by the assumption of coherence and they share two distinct lines). Associating hyperbolic ideal tetrahedra (as above) to each of these hextets therefore gives geometrical tetrahedra whose faces coincide and which fit together smoothly about their shared edge.

The shape of a hyperbolic ideal tetrahedron is determined by a complex shape parameter (see [25]). The shape parameter $z \in \mathbb{C}$ of the hyperbolic tetrahedron associated (by the above procedure) to a given hextet satisfies an equation in cosh of the complex distances between the lines of the hextet. This equation is quite large but it is clearly quadratic in $z$, reflecting the fact that a set of ortholengths only determine a corresponding hextet up to orientation.

Proof of Lemma 14 Let $p \in \mathcal{P}(K) \backslash \mathcal{T}$ be coherent and let $H_{1}, \ldots, H_{n}$ be a coherent realisation of $p$ by hextets, where $H_{j}$ corresponds to the $j^{\text {th }}$ tetrahedron $\triangle_{j}$ of $K$. We can pull the ideal triangulation $K$ back to an ideal triangulation $\widetilde{K}$ of the universal cover $\widetilde{M}$ of $M$ via the covering map $\widetilde{M} \rightarrow M$. Then every tetrahedron, face and edge of $\widetilde{K}$ covers one of the tetrahedra, faces or edges (respectively) of $K$. Let $\widetilde{K}^{(3)}$ denote the set of tetrahedra of $\widetilde{K}$. We will consider a function $H: \widetilde{K}^{(3)} \rightarrow(\mathrm{S} \mathcal{L})^{4}$ which associates a hextet to each tetrahedron of $\widetilde{K}$. This is as an association of an oriented line to each of the four corners of each tetrahedron of $\widetilde{K}$. We require that $H$ satisfies two conditions: (1) if $\widetilde{\triangle} \in \widetilde{K}^{(3)}$ covers the $j^{\text {th }}$ tetrahedron $\triangle_{j}$ of $K$ then the hextet $H(\widetilde{\triangle})$ is isometric to $H_{j}$ and $(2)$ if two tetrahedra $\widetilde{\triangle}, \widetilde{\triangle}^{\prime} \in \widetilde{K}^{(3)}$ share a face $T$ of $\widetilde{K}$ then the right-angled hexagons corresponding to $T$ in $H(\widetilde{\triangle})$ and $H\left(\widetilde{\triangle}^{\prime}\right)$ are identical.

We will show that to each coherent realisation of $p$ by hextets there is a corresponding function $H: \widetilde{K}^{(3)} \rightarrow(\mathrm{S} \mathcal{L})^{4}$ which satisfies the above two conditions, and that this function is unique up to (orientation-preserving) isometry. Before proving this we first show how the existence and rigidity of such a 'skeletal developing map' $H$ proves the lemma.

Given such an $H$, by its uniqueness up to isometry we know that for each deck-transformation $\alpha$ of the covering $\widetilde{M} \rightarrow M$ there is a unique isometry $\rho(\alpha) \in \mathrm{PSL}_{2} \mathbb{C}$ which makes the following diagram

$$
\begin{array}{ccc}
\widetilde{K}^{(3)} & \stackrel{\alpha}{\rightarrow} & \widetilde{K}^{(3)} \\
\downarrow H & & \downarrow H \\
(\mathrm{~S} \mathcal{L})^{4} & \stackrel{\rho(\alpha)}{\rightarrow} & (\mathrm{S} \mathcal{L})^{4}
\end{array}
$$

Algebraic 83 Geometric Topology, Volume 2 (2002) 
commute. Choosing a base-point $\tilde{*} \in \widetilde{M}$ covering $* \in M$ allows us to identify the deck transformations of the covering $\widetilde{M} \rightarrow M$ with $\pi_{1}(M, *)$, and hence $\rho$ becomes a function $\rho: \pi_{1}(M, *) \rightarrow \mathrm{PSL}_{2} \mathbb{C}$. It follows easily from the definition that $\rho: \pi_{1}(M, *) \rightarrow \mathrm{PSL}_{2} \mathbb{C}$ is actually a homomorphism, and it is well-defined up to conjugacy since using a different $H$ or a different base-point $\tilde{*}$ simply has the effect of conjugating $\rho$ by an isometry.

By construction, translates under $\rho\left(\pi_{1}(M, *)\right)$ of the line which is fixed by $\rho\left(\pi_{1}(N, *)\right)$ are simply the lines occurring in the image of $H$, so $\operatorname{Orth}_{K}(\rho)=p$. Up to isometry there are at most $2^{n}$ different realisations of $p$ and so there are at most this many representations $\rho$ with $\operatorname{Orth}_{K}(\rho)=p$ (up to conjugacy). Also, if $p \notin \mathcal{S}$ then up to isometry there are at most two coherent realisations of $p$, and these are the same except that they have opposite orientations. (This is because the orientation of one hextet determines the orientations of all other hextets in a coherent realisation of $p$ whenever all or all but one of the hexagonal faces are non-degenerate, see point (3) of Lemma 10.) These two realisations therefore give rise to functions $H: \widetilde{K}^{(3)} \rightarrow(\mathrm{S} \mathcal{L})^{4}$ which are identical (up to isometry) except that all of their lines are of opposite orientation. Clearly the holonomy representations corresponding to such $H$ are identical.

The rest of the proof is devoted to showing that, corresponding to a coherent realisation $H_{1}, \ldots, H_{n}$ of $p$, there is a function $H: \widetilde{K}^{(3)} \rightarrow(\mathrm{S} \mathcal{L})^{4}$ which satisfies the two conditions listed in the first paragraph of this proof and furthermore that such an $H$ is unique up to (orientation-preserving) isometry.

Dual to $\widetilde{K}$ in $\widetilde{M}$ there is a 2 -dimensional CW-complex $C$ whose underlying space $|C|$ is a deformation retract of $\widetilde{M}$ and so in particular $|C|$ is simply connected. Let $\gamma$ denote a finite sequence $\gamma_{1}, \ldots, \gamma_{m}$ of oriented 1-cells in $C$ so that $\gamma_{i}(1)=\gamma_{i+1}(0)$ for each $i=1, \ldots, m-1$, where $\gamma_{i}(0)$ denotes the start of the 1-cell $\gamma_{i}$ and $\gamma_{i}(1)$ denotes the end of it. Let $x_{0}=\gamma_{1}(0)$ and let $x_{i}=\gamma_{i}(1)$ for each $i=1, \ldots, m$. We think of $\gamma$ as a path in the 1 -skeleton of $C$ from $x_{0}$ to $x_{m}$.

For each $i=1, \ldots, m$, the 0 -cell $x_{i}$ is dual to a tetrahedron $\widetilde{\triangle}_{i}$ (say) of $\widetilde{K}$, and each tetrahedron $\widetilde{\triangle}_{i}$ covers a tetrahedron $\triangle_{j(i)}$ of $K$ (for some $j(i) \in$ $\{1, \ldots, n\})$. Given a choice of hextet $H_{0}^{\prime}$ isometric to $H_{j(0)}$, we define a sequence $H_{0}^{\prime}, \ldots, H_{m}^{\prime}$ of hextets with each $H_{i}^{\prime}$ isometric to $H_{j(i)}$ as follows. Assume that $H_{i-1}^{\prime}$ is defined. Then the 1 -cell $\gamma_{i}$ is dual to some 2 -simplex $\widetilde{T}$ of $\widetilde{K}$ which is contained in both $\widetilde{\triangle}_{i-1}$ and $\widetilde{\triangle}_{i}$. Let $T$ be the 2 -simplex of $K$ covered by $\widetilde{T}$. By the coherence of $p$ we know that the right-angled hexagons in $H_{j(i-1)}$ and $H_{j(i)}$ corresponding to $T$ are isometric. Since $H_{i-1}^{\prime}$ is isometric to $H_{j(i-1)}$ we can define $H_{i}^{\prime}$ as the hextet which is isometric to $H_{j(i)}$ and for which the 
right-angled hexagons in $H_{i-1}^{\prime}$ and $H_{i}^{\prime}$ corresponding to $\widetilde{T}$ are identical. This uniquely determines $H_{i}^{\prime}$ because there is no non-trivial (orientation-preserving) isometry fixing the right-angled hexagon corresponding to $\widetilde{T}$, since no $p_{i}= \pm 1$ and so the hexagon has at least three end-points on $\mathbb{S}_{\infty}^{2}$. Hence to any such path $\gamma$ and any choice $H_{0}^{\prime}$ we have a corresponding sequence $H_{0}^{\prime}, \ldots, H_{m}^{\prime}$ of hextets.

Now, choose some $\widetilde{\triangle} \in \widetilde{K}^{(3)}$ (which covers the $j^{\text {th }}$ tetrahedron $\triangle_{j}$ of $K$, say) and choose a hextet which is isometric to $H_{j}$ and denote it by $H(\widetilde{\triangle})$. Let * be the 0 -cell of $C$ dual to $\widetilde{\triangle}$. Given any other $\widetilde{\triangle}^{\prime} \in \widetilde{K}^{(3)}$, dual to some 0 -cell $x$ of $C$, choose a path $\gamma$ as above with $x_{0}=*$ and $x_{m}=x$. Then form the corresponding sequence $H_{0}^{\prime}, \ldots, H_{m}^{\prime}$ of hextets as defined above with $H_{0}^{\prime}=H(\widetilde{\triangle})$ and define $H\left(\widetilde{\triangle}^{\prime}\right)$ to be $H_{m}^{\prime}$.

To see that this definition is independent of the path from $*$ to $x$, note that any two such paths are homotopic in $|C|$. It therefore suffices to show that the holonomy around the boundary of any of the 2-cells of $C$ is trivial, i.e. that if $\gamma$ is a path tracing once around a 2-cell of $C$ then in the corresponding sequence $H_{0}^{\prime}, \ldots, H_{m}^{\prime}$ of hextets defined above, $H_{0}^{\prime}=H_{m}^{\prime}$. But for such a $\gamma$, all of the tetrahedra $\widetilde{\triangle}_{0}, \ldots, \widetilde{\triangle}_{m}$ share an edge in $\widetilde{K}$ and so by construction all of the hextets $H_{0}^{\prime}, \ldots, H_{m}^{\prime}$ have two lines in common. So the hextets $H_{0}^{\prime}$ and $H_{m}^{\prime}$ share two lines and are isometric (since $\widetilde{\triangle}_{0}=\widetilde{\triangle}_{m}$ ). Then since there are no non-trivial orientation-preserving isometries fixing two distinct ${ }^{12}$ lines in $\mathbb{H}^{3}$, $H_{0}^{\prime}=H_{m}^{\prime}$ as required.

It is clear that $H: \widetilde{K}^{(3)} \rightarrow(\mathrm{S} \mathcal{L})^{4}$ as constructed satisfies the two defining conditions given in the first paragraph of this proof, and conversely any such $H$ can be constructed in this way. Hence the existence and rigidity of $H$ is proved.

Non-coherent ortholengths correspond to representations into $\mathrm{PSL}_{2} \mathbb{C}$ of the fundamental groups of finite-sheeted covers or branched covers of $M$, branched over the edges of $K$. Hence non-coherent ortholengths seem to be more related to the ideal triangulation $K$ than to intrinsic properties of the 3 -manifold $M$.

We next show that the property of coherence is locally constant on the complement of a certain subvariety of $\mathcal{P}(K)$. This proof is a deformation argument in which we make use of the fact that in some sense there exists a continuous local parameterisation of hextets by their ortholengths. We pause now briefly to give a precise description of this local parameterisation.

\footnotetext{
${ }^{12}$ Since no $p_{i}= \pm 1$, the two lines have more than three end-points on $\mathbb{S}_{\infty}^{2}$.
} 
Define the set of reduced hextets $\mathcal{R H}_{4}$ to be

$$
\mathcal{R H}_{4} \stackrel{\text { def }}{=}\left\{\left(l_{1}, \ldots, l_{4}\right) \in(\mathrm{S} \mathcal{L})^{4} \mid l_{1}=\left[\begin{array}{cc}
i & 0 \\
0 & -i
\end{array}\right], l_{2}=\left[\begin{array}{cc}
a & i-a \\
i+a & -a
\end{array}\right], a \neq \pm i \in \mathbb{C}\right\},
$$

i.e. the set of hextets whose lines $l_{1}, \ldots, l_{4}$ are in standard position so that $l_{1}$ has ordered end-points $(0, \infty)$ and $l_{2}$ has ordered end-points $(b, 1)$ for some $b \in \mathbb{S}_{\infty}^{2}$ not equal to 0,1 or $\infty$. Note that any hextet $l_{1}, \ldots, l_{4}$ with $<l_{1}, l_{2}>\neq \pm 1$ is isometric to exactly one of the hextets in $\mathcal{R H}_{4}$. Now, let

$$
\mathcal{P}(\triangle)=\left\{\left(x_{12}, x_{13}, x_{14}, x_{23}, x_{24}, x_{34}\right) \in \mathbb{C}^{6} \mid x_{12} \neq \pm 1 \text { and } \operatorname{det} X=0\right\}
$$

where

$$
X \stackrel{\text { def }}{=}\left[\begin{array}{cccc}
1 & x_{12} & x_{13} & x_{14} \\
x_{12} & 1 & x_{23} & x_{24} \\
x_{13} & x_{23} & 1 & x_{34} \\
x_{14} & x_{24} & x_{34} & 1
\end{array}\right]
$$

and let $D_{\triangle}: \mathcal{R H}_{4} \rightarrow \mathcal{P}(\triangle)$ be the quadratic map given in the above coordinates by $x_{i j}=<l_{i}, l_{j}>$. By Lemma $3, D_{\triangle}$ takes a hextet and gives us cosh of the ortholengths between the hextet's four lines.

Now, by Corollary 8 and Lemma $10, D_{\triangle}$ is onto and it is two-to-one everywhere except on the set of degenerate hextets where $D_{\triangle}$ is instead one-to-one. By Lemma 10 again, the degenerate hextets are the pre-image of those elements of $\mathcal{P}(\triangle)$ for which $\operatorname{rank}(X)=2$. The restriction of $D_{\triangle}$ to the non-degenerate hextets is a double cover onto the elements of $\mathcal{P}(\triangle)$ for which $\operatorname{rank}(X)=3$. The deck-transformation for this cover is the involution $\sigma: \mathcal{R H}_{4} \rightarrow \mathcal{R} \mathcal{H}_{4}$ given by $\sigma:\left(l_{1}, l_{2}, l_{3}, l_{4}\right) \mapsto\left(l_{1}, l_{2}, f_{a}\left(l_{3}\right), f_{a}\left(l_{4}\right)\right)$ where

$$
f_{a}\left(\left[\begin{array}{cc}
u & v \\
w & -u
\end{array}\right]\right)=\left[\begin{array}{cc}
u & \frac{i-a}{i+a} w \\
\frac{i+a}{i-a} v & -u
\end{array}\right]
$$

and $a \neq \pm i$ is determined by $l_{2}=\left[\begin{array}{cc}a & i-a \\ i+a & -a\end{array}\right]$.

Similarly, we define the set of reduced right-angled hexagons to be

$$
\mathcal{R H}_{3} \stackrel{\text { def }}{=}\left\{\left(l_{1}, l_{2}, l_{3}\right) \in(\mathrm{S} \mathcal{L})^{3} \mid l_{1}=\left[\begin{array}{cc}
i & 0 \\
0 & -i
\end{array}\right], l_{2}=\left[\begin{array}{cc}
a & i-a \\
i+a & -a
\end{array}\right], a \neq \pm i \in \mathbb{C}\right\},
$$

and define

$$
\mathcal{P}(F)=\left\{\left(x_{12}, x_{13}, x_{23}\right) \in \mathbb{C}^{3} \mid x_{12} \neq \pm 1\right\}
$$

and let $D_{F}: \mathcal{R H}_{3} \rightarrow \mathcal{P}(F)$ be the map given in the above co-ordinates by $x_{i j}=<l_{i}, l_{j}>$. As above, $D_{F}$ restricts to a double covering from the set of 
non-degenerate right-angled hexagons to the subset of $\mathcal{P}(F)$ for which

$$
\operatorname{det}\left[\begin{array}{ccc}
1 & x_{12} & x_{13} \\
x_{12} & 1 & x_{23} \\
x_{13} & x_{23} & 1
\end{array}\right] \neq 0
$$

Lemma 15 The property of coherence is locally constant on $\mathcal{P}(K) \backslash(\mathcal{S} \cup \mathcal{T})$.

Proof Let $a$ be a point of $\mathcal{P}(K) \backslash(\mathcal{S} \cup \mathcal{T})$ and for each $i=1, \ldots, n$, choose a numbering from 1 to 4 for the vertices of the $i^{\text {th }}$ tetrahedron $\triangle_{i}$ of $K$. Then we have a projection $\pi_{i}: \mathcal{P}(K) \rightarrow \mathcal{P}(\triangle)$ essentially given by ignoring the edge parameters which do not appear on the edges of $\triangle_{i}$. We know that $\pi_{i}(a)$ is a smooth point of the hypersurface $\mathcal{P}(\triangle) \subseteq \mathbb{C}^{6}$, since $D_{\triangle}$ is a local diffeomorphism onto a neighbourhood of $\pi_{i}(a)$ (since $\pi_{i}(a)$ corresponds to a non-degenerate hextet) and $\mathcal{R H}_{4}$ is easily seen to be smooth everywhere. Let $V_{i} \subseteq \mathcal{P}(\triangle)$ be a neighbourhood of $\pi_{i}(a)$ which is diffeomorphic to $\mathbb{C}^{5}$ and which is disjoint from the hypersurface given by the equation $\operatorname{rank}(X)=2$, where $X$ is as defined in (4). (This is possible since $a \notin \mathcal{S}$ and hence $\pi_{i}(a)$ corresponds to a non-degenerate hextet.) Let $U$ be a connected neighbourhood of $a$ in $\mathcal{P}(K) \backslash(\mathcal{S} \cup \mathcal{T})$ which is contained in $\pi_{i}^{-1}\left(V_{i}\right)$ for each $i=1, \ldots, n$.

Now, suppose that there exists some $p \in U$ which is coherent, say with a coherent realisation $H_{1}^{p}, \ldots, H_{n}^{p} \in \mathcal{R} \mathcal{H}_{4}$, and let $q$ be any other point of $U$. For each $i=1, \ldots, n$, the image of $U$ under $\pi_{i}: \mathcal{P}(K) \rightarrow \mathcal{P}(\triangle)$ is contained in the path-connected, locally path-connected and simply connected set $V_{i}$. Also, the restriction of $D_{\triangle}: \mathcal{R H}_{4} \rightarrow \mathcal{P}(\triangle)$ to $D_{\triangle}{ }^{-1}\left(V_{i}\right)$ is a covering projection. Hence by the lifting property of coverings (see [4]) there is a unique lift $\tilde{\pi}_{i}: U \rightarrow \mathcal{R} \mathcal{H}_{4}$ of the restriction $\pi_{i} \mid U$ of $\pi_{i}$ to $U$ for which $\tilde{\pi}_{i}(p)=H_{i}^{p}$. We define $H_{i}^{q}$ to be the hextet $\tilde{\pi}_{i}(q)$.

Now, let $F$ be any face of $K$ and suppose that $F$ is contained in tetrahedra $\triangle_{i}$ and $\triangle_{j}$ of $K$. If we choose a numbering from 1 to 3 for the vertices of $F$ then we can define several projection maps. Firstly a projection $\pi_{F}: \mathcal{P}(K) \rightarrow$ $\mathcal{P}(F)$ essentially given by forgetting all ortholength parameters except those associated to the edges of $F$. Also, a map $\tau_{i}: \mathcal{R H}_{4} \rightarrow \mathcal{R} \mathcal{H}_{3}$ which first forgets the line of $H_{i}^{q} \in \mathcal{R H}_{4}$ corresponding to the vertex of $\triangle_{i}$ not contained in $F$ and which then acts by an (orientation-preserving) isometry to bring the resulting right-angled hexagon into standard position. Similarly we have a map $\tau_{j}: \mathcal{R H}_{4} \rightarrow \mathcal{R} \mathcal{H}_{3}$.

Now, if the right-angled hexagons corresponding to $F$ in $H_{i}^{q}$ and $H_{j}^{q}$ are degenerate then they are isometric by Lemma 10. Hence we can restrict our attention 
to the set $U^{\prime}$ consisting of $q \in U$ so that $H_{i}^{q}$ and $H_{j}^{q}$ are non-degenerate. Then $\tau_{i} \circ \tilde{\pi}_{i}: U^{\prime} \rightarrow \mathcal{R} \mathcal{H}_{3}$ and $\tau_{j} \circ \tilde{\pi}_{j}: U^{\prime} \rightarrow \mathcal{R} \mathcal{H}_{3}$ are both lifts of the restriction $\pi_{F} \mid U^{\prime}$ of $\pi_{F}$ to $U^{\prime}$ and they agree at $p$. Hence by the uniqueness of lifts to covering spaces (see [4]), $\tau_{i} \circ \tilde{\pi}_{i}=\tau_{j} \circ \tilde{\pi}_{j}$ and so the right-angled hexagons corresponding to $F$ in $H_{i}^{q}$ and $H_{j}^{q}$ are again isometric.

Lemma 16 The limit of a sequence of coherent points in $\mathcal{P}(K)$ is coherent.

Proof This lemma follows from the fact that the inverse images of compact sets in $\mathcal{P}(\triangle)$ under $D_{\triangle}: \mathcal{R H}_{4} \rightarrow \mathcal{P}(\triangle)$ are compact.

A rational map between two irreducible complex varieties which is generically ${ }^{13}$ defined, injective and onto has a rational inverse and is called a birational equivalence (see [14, p.77]). Hence Lemmas 14, 15 and 16 combine to give the following theorem.

Theorem 17 Let $C$ be an irreducible component of $X(M)$ on which Orth $_{K}$ is generically defined and suppose that the set-theoretic image of $C$ under the rational map Orth $_{K}: X(M) \rightarrow \mathcal{P}(K)$ contains some point $p=\left(p_{1}, \ldots, p_{n}\right) \in$ $\mathcal{P}(K)$ for which $p \notin \mathcal{S}$ (see equation (3)) and no $p_{i}= \pm 1$. Then Orth $_{K}$ restricts to a birational equivalence between $C$ and an irreducible component of $\mathcal{P}(K)$.

Let $H o l: \mathcal{H}(M) \rightarrow X(M)$ be the map which takes a hyperbolic structure and returns its holonomy representation modulo conjugacy. Then as in Section 3, the ortholength invariant of an incomplete hyperbolic structure $M_{0} \in \mathcal{H}(M)$ is $\operatorname{Orth}_{K} \circ \operatorname{Hol}\left(M_{0}\right)$. Recall also that we say that an incomplete hyperbolic structure on $M$ corresponds to a (topological) Dehn filling if the metric completion of $M$ is a closed manifold (homeomorphic to a topological Dehn filling of $M$ ) with a smooth hyperbolic structure.

Theorem 18 Let $M_{0} \in \mathcal{H}(M)$ be an incomplete hyperbolic structure whose ortholength invariant $p=\left(p_{1}, \ldots, p_{n}\right) \in \mathcal{P}(K)$ is not contained in $\mathcal{S}$ (see equation (3)) and which has no $p_{i}= \pm 1$. Then:

- If $M_{0}$ corresponds to a (topological) Dehn filling of $M$ then $M_{0}$ is uniquely determined by $p$.

\footnotetext{
${ }^{13} \mathrm{~A}$ property is generically satisfied on a variety if it is true on the complement of a proper subvariety.
} 
- The ortholength invariant Orth $_{K}: X(M) \rightarrow \mathcal{P}(K)$ restricts to a diffeomorphism from a neighbourhood of $\operatorname{Hol}\left(M_{0}\right)$ in $X(M)$ to a neighbourhood of $p$ in $\mathcal{P}(K)$.

The holonomy map Hol is a local homeomorphism (e.g. see [25, §5.2] or [11]) so we can endow $\mathcal{H}(M)$ with a smooth structure coming from $X(M)$. Then the second point of this theorem says that (generically) the ortholength invariant smoothly locally parameterises incomplete hyperbolic structures on $M$.

Proof of Theorem 18 A hyperbolic structure which corresponds to a topological Dehn filling of $M$ is determined by its holonomy representation. This is a 'folk-lore' result which is true essentially because, in this case, the image of the holonomy representation is a discrete Kleinian group, and its action on $\mathbb{H}^{3}$ has a quotient isometric to the metric completion of $M$. But by Lemma 14, the ortholength invariant $p$ determines the holonomy representation whenever $p \notin \mathcal{S}$ and no $p_{i}= \pm 1$. This proves the first point of the corollary.

Now, $\operatorname{Orth}_{K}$ is defined on every representation which arises as the holonomy of one of the hyperbolic structures of $\mathcal{H}(M)$ (see Section 3). Then since the conditions (2) are open, Orth $_{K}$ is defined in a neighbourhood of $\operatorname{Hol}\left(M_{0}\right)$. From the formula of Proposition 12 it is clear that Orth $_{K}$ is smooth in this neighbourhood.

On the other hand, $p$ is coherent by the construction used to define the ortholength invariant in Section 3. By Lemma 15 this implies that all ortholength parameters in a neighbourhood of $p$ are also coherent. Hence by Lemma 14 there is a local inverse to $\operatorname{Orth}_{K}$ defined in a neighbourhood of $p$. This inverse is smooth by the construction given in Lemma 14, since the representation given there is determined by a finite portion of the skeletal developing map $H$ and the hextets used to build this map all vary smoothly with $p$. This last result follows from the discussion preceding Lemma 15 and the fact that if $p \notin \mathcal{S}$ then the hextets in a realisation of $p$ are all non-degenerate.

Theorems 17 and 18 are vacuous unless there exists some incomplete hyperbolic structure with ortholength invariant $p$ such that $p \notin \mathcal{S}$ and no $p_{i}= \pm 1$. This will probably be the case whenever the edges of $K$ are homotopically non-trivial, however until such a general result can be established we have the following lemma.

Lemma 19 If the ideal triangulation $K$ is Epstein-Penner's [9] ideal celldecomposition of $M$ then there exists an incomplete hyperbolic structure with ortholength invariant $p$ such that $p \notin \mathcal{S}$ and no $p_{i}= \pm 1$. 
The full proof of this lemma relies on the notion of a 'tube domain' (see Section 6 and [8, Lemma 4.7]) so we content ourselves here with a sketch.

Sketch of a proof of Lemma 19 Corresponding to the complete hyperbolic structure on $M$ there is a 2-complex in $M$ which is the image of the boundary of the Ford domain of $M$ under the face-pairing identifications. This Ford 2-complex is dual to $K$ (see [9]). Let $\bar{M}$ be a (topological) Dehn filling of $M$ with very large Dehn surgery co-ordinates and let $\Sigma$ be the added 'core' geodesic of $\bar{M}$, hence $\bar{M}$ is hyperbolic and $M=\bar{M} \backslash \Sigma$. The cut-locus of $\Sigma$ in $\bar{M}$ is a 2-complex which (for very large Dehn fillings) is a small perturbation of the Ford 2-complex, and so in particular this cut-locus is also topologically dual to $K$ (see [8]). Now, the pre-image of $\Sigma$ under a (fixed) covering isometry $\mathbb{H}^{3} \rightarrow \bar{M}$ is a collection of disjoint lines in $\mathbb{H}^{3}$. If $F_{j}$ is a 2 -simplex of $K$ and $\widetilde{F}_{j}$ is a lift to $\mathbb{H}^{3}$ then each corner of $\widetilde{F}_{j}$ determines one of these lines. The complex distances between these three distinguished lines are given by the three ortholength parameters $p_{i}$ attached to the corresponding edges of $F_{j}$, where $p=\left(p_{1}, \ldots, p_{n}\right)$ is the ortholength invariant of the hyperbolic structure on $M$ induced from $M \hookrightarrow \bar{M}$. Then the set of points of $\mathbb{H}^{3}$ which are equidistant from all three lines simultaneously is non-empty, since this set contains a lift of a certain part of the cut-locus of $\Sigma$, namely the 1-cell dual to $F_{j}$.

But if $p$ lies in $\mathcal{S}_{j}$ then this is a contradiction. In this case, the three lines corresponding to the corners of $\widetilde{F}_{j}$ have a common perpendicular line $n$ (by Lemma 10). Since $\bar{M}$ is a large Dehn filling, all of its ortholengths will be very large ${ }^{14}$ and so the distances between these three lines are also large (and no $p_{i}= \pm 1$ ). This implies that the locus of points equidistant from any pair of lines is close to the plane which is perpendicular to $n$ and lies mid-way between the two lines. Hence there can be no point of $\mathbb{H}^{3}$ which is equidistant from all three lines simultaneously.

\section{$5 \quad$ Examples}

In this section we describe the ortholength invariant Orth $_{K}: X(M) \rightarrow \mathcal{P}(K)$ for the figure- 8 knot complement $M$.

Let $M$ be the complement in $\mathbb{S}^{3}$ of the figure-8 knot (see Figure 3). A result of Epstein and Penner (see [9]) says that every 1-cusped hyperbolic 3-manifold has a canonical ideal cell-decomposition. For the figure- 8 knot complement $M$, this

\footnotetext{
${ }^{14}$ For large Dehn fillings the tube radius is large, see [25].
} 


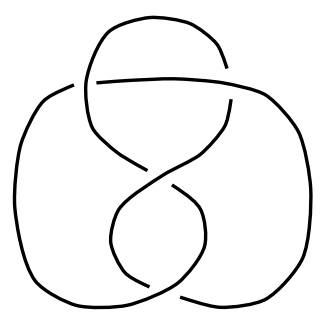

Figure 3: The figure- 8 knot

cell-decomposition is the ideal triangulation $K$ shown $^{15}$ in Figure 4 (see [27]). This ideal triangulation for the figure- 8 knot complement was first described by Thurston [25].

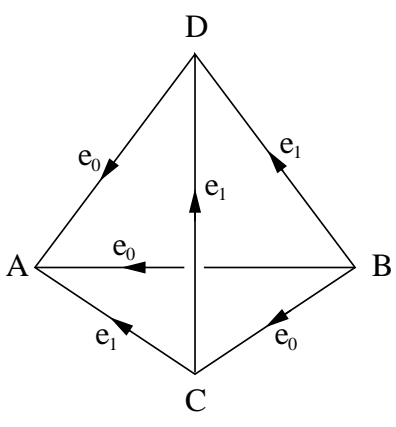

(a) Tetrahedron 0

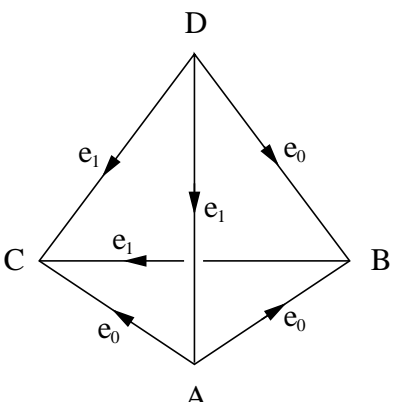

(b) Tetrahedron 1

Figure 4: The canonical ideal triangulation $K$ for the figure-8 knot complement

The ideal triangulation $K$ has two edge classes, $e_{0}$ and $e_{1}$. Let $p_{0}$ be the ortholength parameter corresponding to edge class $e_{0}$ and let $p_{1}$ correspond to $e_{1}$. Then the hextet equation (see Definition 11) of tetrahedron 0 is

$$
\begin{aligned}
0=\operatorname{det} & {\left[\begin{array}{cccc}
1 & p_{0} & p_{1} & p_{1} \\
p_{0} & 1 & p_{1} & p_{0} \\
p_{1} & p_{1} & 1 & p_{0} \\
p_{1} & p_{0} & p_{0} & 1
\end{array}\right] } \\
= & 1-3 p_{0}^{2}-3 p_{1}^{2}+4 p_{0} p_{1}^{2}+4 p_{0}^{2} p_{1}+p_{0}^{4}-2 p_{0}^{3} p_{1}-p_{0}^{2} p_{1}^{2}-2 p_{0} p_{1}^{3}+p_{1}^{4} \\
= & \frac{1}{4}\left(p_{0}^{2}+p_{1}^{2}+p_{0} p_{1}-p_{0}-p_{1}-1\right)\left(2 p_{1}-3 p_{0}+1+\sqrt{5}\left(p_{0}-1\right)\right) \\
& \times\left(2 p_{1}-3 p_{0}+1-\sqrt{5}\left(p_{0}-1\right)\right) .
\end{aligned}
$$

\footnotetext{
${ }^{15}$ The faces of the tetrahedra shown in Figure 4 are identified in pairs giving faceclasses A,B,C and D. The class of each face is written at the vertex opposite the face.
} 
The hextet equation of tetrahedron 1 is identical, so $\mathcal{P}(K) \subseteq \mathbb{C}^{2}$ is the plane algebraic curve given by equation (5). From (5) we see that $\mathcal{P}(K)$ is the union of a conic (whose projective completion is topologically a smooth sphere) and two lines.

Following the notation of Section 3 we take $N \subseteq M$ to be a closed tubular neighbourhood of the figure- 8 knot minus the knot itself. Then the generators of the presentation of $\pi_{1}(M, *)$ based on $K$ (see the discussion preceding Proposition 12) are as shown in Figure 5.

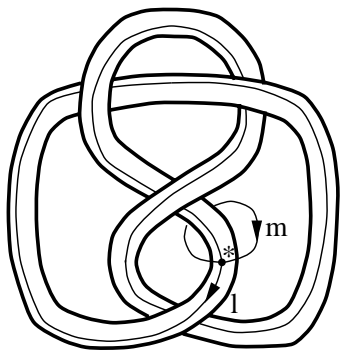

(a) The generators for $\pi_{1}(\partial N, *)$

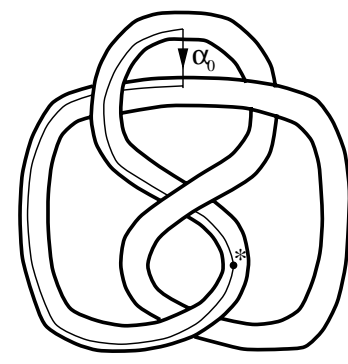

(b) The generator $\alpha_{0}$

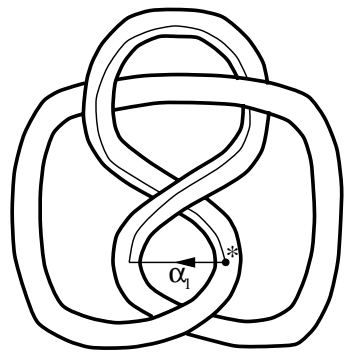

(c) The generator $\alpha_{1}$

Figure 5: The loops corresponding to the generators of the presentation for $\pi_{1}(M, *)$ based on $K$

Now, let $t_{1}=l \alpha_{1}^{-1} m$ and $t_{2}=m$. These are the generators of a presentation

$$
\pi_{1}(M, *)=<t_{1}, t_{2} \mid\left[t_{2}^{-1}, t_{1}\right] t_{2}^{-1}=t_{1}^{-1}\left[t_{2}^{-1}, t_{1}\right]>
$$

which is derived from a Wirtinger presentation for $\pi_{1}(M, *)$. We define two complex algebraic functions $X$ and $Y$ on the space of representations of $\pi_{1}(M, *)$ into $\mathrm{SL}_{2} \mathbb{C}$ by

$$
X(\tilde{\rho})=\operatorname{tr}\left(\tilde{\rho}\left(t_{1}\right)\right)=\operatorname{tr}\left(\tilde{\rho}\left(t_{2}\right)\right) \text { and } Y(\tilde{\rho})=\operatorname{tr}\left(\tilde{\rho}\left(t_{1} t_{2}\right)\right)
$$

for any representation $\tilde{\rho}: \pi_{1}(M, *) \rightarrow \mathrm{SL}_{2} \mathbb{C}$. These functions are clearly conjugacy invariant, so they descend to functions (also denoted $X$ and $Y$ ) on the $\mathrm{SL}_{2} \mathbb{C}$-character variety ${ }^{16}$. In fact, $X$ and $Y$ are the co-ordinate functions of an embedding of the $\mathrm{SL}_{2} \mathbb{C}$-character variety in $\mathbb{C}^{2}$ as the complex curve given by

$$
0=\left(X^{2}-Y-2\right)\left(X^{2} Y-2 X^{2}-Y^{2}+Y+1\right)
$$

\footnotetext{
${ }^{16}$ Similarly to the $\mathrm{PSL}_{2} \mathbb{C}$-character variety, the $\mathrm{SL}_{2} \mathbb{C}$-character variety of $M$ is defined to be the algebro-geometric quotient of the space of representations $\pi_{1}(M, *) \rightarrow$ $\mathrm{SL}_{2} \mathbb{C}$ by the conjugacy action of $\mathrm{SL}_{2} \mathbb{C}$, as defined in Culler-Shalen [7].
} 
(see González-Acuña and Montesinos-Amilibia's paper [12]).

Now, from the presentation (6) above it is clear that each representation $\rho$ : $\pi_{1}(M, *) \rightarrow \mathrm{PSL}_{2} \mathbb{C}$ lifts to exactly two representation $\pm \tilde{\rho}: \pi_{1}(M, *) \rightarrow \mathrm{SL}_{2} \mathbb{C}$. Hence there are well-defined algebraic functions $U$ and $V$ defined on the $\mathrm{PSL}_{2} \mathbb{C}$-character variety $X(M)$ by

$$
U(\rho)=X^{2}(\tilde{\rho}) \text { and } V(\rho)=X^{2}(\tilde{\rho})-Y(\tilde{\rho}),
$$

i.e. $U$ and $V$ are elements of the co-ordinate ring of $X(M)$. By (7), these functions define an embedding of $X(M)$ into $\mathbb{C}^{2}$ as the complex curve given by the equation

$$
0=(V-2)\left(V^{2}-U V+V+U-1\right) .
$$

Hence $X(M)$ is composed of two irreducible components, one being the line $V=2$ and the other being the rational curve

$$
U=\frac{V^{2}+V-1}{V-1} .
$$

The line $V=2$ corresponds to reducible representations (i.e. those which fix a point on $\mathbb{S}_{\infty}^{2}$ ) and the rational curve (9) corresponds to conjugacy classes of irreducible representations (see [12]).

We can now calculate $\operatorname{Orth}_{K}: X(M) \rightarrow \mathcal{P}(K)$ using the formula given in Proposition 12. The conditions (2) are satisfied at all points of $X(M)$ except the points with $(U, V)$ equal to $(4,2)$ or $(4,1-\zeta)$, where $\zeta \in \mathbb{C}$ is a nontrivial cube root of unity. (These points correspond to the trivial representation and to the two orientation-preserving conjugacy classes of discrete and faithful representations.) Since the line $V=2$ corresponds to reducible representations (see [12]) Orth $_{K}$ takes the whole line to the point $(1,1)$. On the curve (9), Proposition 12 implies that

$$
\operatorname{Orth}_{K}(U, V)=\frac{1}{V^{2}-3 V+3}\left(-V^{2}+3 V-1, V^{2}-V-1\right)
$$

From this expression it is easy to check that the image of $\operatorname{Orth}_{K}$ lies inside the conic component of $\mathcal{P}(K)$. From (10) and Figure 4, the ortholength invariant $\operatorname{Orth}_{K}(U, V)$ lies in $\mathcal{S}$ whenever

$$
0=(V-2)^{2}\left(V^{2}+V-1\right) \text { and } 0=(V-2)^{2}(V-1)^{2}\left(V^{2}+V-1\right),
$$

where $(U, V) \in X(M)$ belongs to the curve (9), i.e. $\operatorname{Orth}_{K}(U, V) \in \mathcal{S}$ when $V$ equals 2 or $(-1 \pm \sqrt{5}) / 2$. Also, a simple calculation shows that $\operatorname{Orth}_{K}(U, V) \in$ $\mathcal{P}(K) \subseteq \mathbb{C}^{2}$ has one or both co-ordinates equal to \pm 1 if and only if $V$ is 1 or 2 . Hence by Theorem 17, the image of Orth $_{K}$ is dense in the conic component of 
$\mathcal{P}(K)$ and Orth $_{K}$ restricts to a birational equivalence between this component and the curve (9). A simple calculation shows that the inverse of Orth $_{K}$ on this curve is given by $\left(p_{0}, p_{1}\right) \mapsto(U, V)$, where

$$
V=\frac{2 p_{0}+p_{1}+1}{p_{0}+1}
$$

and $U$ is given by the equation (9).

\section{$6 \quad$ Future applications}

The work presented in this paper arose out of an attempt to construct families of incomplete hyperbolic structures on a cusped 3-manifold $M$ and to estimate the boundary of the deformation space $\mathcal{H}(M)$ in Dehn surgery co-ordinates.

The connection between the ortholength invariant and these two problems is the concept of a tube domain (see [8]) which comes from collaborative work with Craig D. Hodgson. A tube domain can be defined for any (incomplete) hyperbolic structure in $\mathcal{H}(M)$, but the description is simplest for those hyperbolic structures which correspond to topological Dehn fillings of $M$. If $\bar{M}$ is a hyperbolic Dehn filling of $M$ then there is a covering isometry $\pi: \mathbb{H}^{3} \rightarrow \bar{M}$ and a distinguished simple closed geodesic $\Sigma$ in $\bar{M}$ so that $\bar{M} \backslash \Sigma$ is diffeomorphic to $M$. We define the tube domain $\mathcal{D}$ of the Dehn filling to be (the closure of) the set $\widetilde{\mathcal{D}}$ of points of $\mathbb{H}^{3}$ which are closer to a line $\widetilde{\Sigma} \subseteq \pi^{-1}(\Sigma)$ than to any other line of $\pi^{-1}(\Sigma)$, modulo the deck-transformations which preserve $\widetilde{\Sigma}$. There is a surjective map $\mathcal{D} \rightarrow \bar{M}$ which makes the diagram

$$
\begin{array}{llll}
\widetilde{\mathcal{D}} & \hookrightarrow & \mathbb{H}^{3} \\
\downarrow & & \downarrow \pi \\
\mathcal{D} & \rightarrow & \frac{\downarrow}{M}
\end{array}
$$

commute. The tube domain $\mathcal{D}$ is diffeomorphic to a solid torus and its boundary is broken into (non-planar) faces which the map $\mathcal{D} \rightarrow \bar{M}$ isometrically identifies in pairs.

On the other hand, the ortholength invariant of the hyperbolic structure $\bar{M} \backslash \Sigma$ on $M$ determines the set of lines $\pi^{-1}(\Sigma)$ up to isometry (as detailed in the proof of Lemma 14). These in turn determine the corresponding tube domain $\mathcal{D}$ and its face-pairing isometries, and so give us back the original hyperbolic structure $\bar{M} \backslash \Sigma$ on $M$. This gives us a method of constructing hyperbolic structures via tube domains which can be generalised to calculate hyperbolic structures with more general Dehn surgery-type singularities, including hyperbolic conemanifolds whose singular sets are simple closed geodesics. This approach has 
been automated by Goodman-Hodgson in a computer program [13] called Tube. Tube naturally lends itself to calculating the tube radius and it has been used to calculate hyperbolic structures which SnapPea [28] fails to compute. For instance, Tube has calculated a hyperbolic structure for m004(4,0) (the hyperbolic cone-manifold with cone-angles $\pi / 2$ along the figure- 8 knot) and for m007 $(3,1)$ (the manifold of third lowest volume on SnapPea's closed census) while SnapPea fails to compute a hyperbolic structure for m004 $(4,0)$ and there is no known positively oriented tetrahedral decomposition of m007(3,1). Also, the cone-manifolds over the figure- 8 knot complement with cone angle strictly less than $2 \pi / 3$ all have hyperbolic structures (see [15] and also [16, §29]) but for cone angles bigger than $2 \pi / 4.767 \ldots$ SnapPea's tetrahedral construction fails. In fact, Choi [5] has shown that there cannot exist a positively-oriented hyperbolic ideal triangulation for the cone-manifold over the figure- 8 knot complement when cone-angles are $2 \pi / 4.767 \ldots$ (i.e. when one of the tetrahedra of the canonical ideal triangulation flattens out).

The second problem which motivates the study of the ortholength invariant is the estimation of the boundary of $\mathcal{H}(M)$. The ortholength invariant seems especially suited to this task because of its close relationship with the tube radius and because of some suggestive connections between the tube radius and the degeneration of hyperbolic structures on $M$ (e.g. see Kojima [19] and Hodgson-Kerckhoff [18], [17]). For a given (incomplete) hyperbolic structure $M_{0} \in \mathcal{H}(M)$, there is a (topological) ideal triangulation $K_{0}$ so that the tube radius of $M_{0}$ is given in terms of $\operatorname{Orth}_{K_{0}}\left(M_{0}\right)$. In particular, the tube radius of $M_{0}$ will be non-zero whenever all co-ordinates of $\operatorname{Orth}_{K_{0}}\left(M_{0}\right)$ lie in $\mathbb{C} \backslash[-1,1]$.

Each edge of this special ideal triangulation $K_{0}$ corresponds to a pair of faces of the tube domain of $M_{0}$. The combinatorics of the tube domains and hence of the corresponding ideal triangulations $K_{0}$ are locally constant in $\mathcal{H}(M)$ and also at the complete structure (see $[8, \S 4.5]$ ). Since $\mathcal{H}(M)$ is non-compact near its boundary, it is conceivable that infinitely many inequivalent triangulations $K_{0}$ may arise as $M_{0}$ varies over $\mathcal{H}(M)$. However, we conjecture that this in not the case, i.e. for any given $M$ only a finite number $K_{1}, \ldots, K_{m}$ of (topological) ideal triangulations are needed to compute the tube radii of the incomplete hyperbolic structures in $\mathcal{H}(M)$. For our irreducible manifold $M$, the conjecture of [18] reduces to the assertion that a family of hyperbolic structures degenerates if and only if their tube radii approach zero. This motivates the following conjecture.

Conjecture 20 Let $M$ be the underlying smooth manifold of an orientable, 1cusped hyperbolic 3-manifold of finite volume. Then there are a finite number 
of (topological) ideal triangulations $K_{1}, \ldots, K_{m}$ of $M$ so that a sequence of (incomplete) hyperbolic structures in $\mathcal{H}(M)$ has a limit in $\mathcal{H}(M)$ if and only if the corresponding $m$ sequences of ortholength invariants with respect to the ideal triangulations $K_{1}, \ldots, K_{m}$ all have limits with co-ordinates $\cosh d_{i}$ in $\mathbb{C} \backslash[-1,1]$

For instance, when $M$ is the figure- 8 knot complement, computational evidence suggests that this conjecture is true with $m=1$ and $K_{1}$ the ideal triangulation of Section 5. More specifically, in Dehn surgery co-ordinates the zero-volume set (which in this case is conjectured to be the boundary of $\mathcal{H}(M)$, see [25], [16]) appears to coincide with the frontier of the set of hyperbolic structures with $p_{0}, p_{1} \notin[-1,1] \subseteq \mathbb{C}$, where $\left(p_{0}, p_{1}\right)$ is the ortholength invariant of Section 5 .

Practical conditions to determine when the tube domain construction fails may provide rigorous estimates about the boundary of $\mathcal{H}(M)$, especially in special cases such as the figure- 8 knot complement. Analogies between the construction (in practice) of Dirichlet domains and the construction of tube domains suggest possible necessary conditions for the failure of the tube domain construction though no useful estimates have emerged from this approach so far.

We can also attempt to use ortho-angles (which are dual to the ortholengths) to parameterise $\mathcal{H}(M)$. The ortho-angles are more directly related to the Dehn surgery co-ordinates of $\mathcal{H}(M)$ than the ortholengths and they are very wellbehaved near the complete structure. This makes the ortho-angle parameterisation of $\mathcal{H}(M)$ quite a promising line of research.

Finally we note that even though we have restricted our attention in this paper to single-cusped 3-manifolds, the main definitions and proofs ${ }^{17}$ trivially extend to 3 -manifolds with multiple cusps.

\section{References}

[1] R. Benedetti and C. Petronio, Lectures on hyperbolic geometry, Springer, Berlin, 1992.

[2] M. Boileau and J. Porti, Geometrization of 3-orbifolds of cyclic type, Asterisque, 272, 2001.

[3] S. Boyer and X. Zhang, On Culler-Shalen seminorms and Dehn filling, Ann. of Math. (2) 148 (1998), no. 3, 737-801.

\footnotetext{
${ }^{17}$ In particular, Theorems 17 and 18 are true in the case of multiple cusps.
} 
[4] G. E. Bredon, Topology and Geometry, GTM 139, Springer, New York, 1993.

[5] Young-Eun Choi, Positively oriented ideal triangulations on hyperbolic 3manifolds, Ph.D. thesis, Stanford, 2000.

[6] D. Cooper, C. Hodgson and S. Kerckhoff, Three-dimensional Orbifolds and Cone-Manifolds,

Memoirs of the Mathematical Society of Japan, Vol 5, 2000.

[7] M. Culler and P. B. Shalen, Varieties of group representations and splittings of 3-manifolds, Ann. of Math. 117 (1983), 109-146.

[8] J. G. Dowty, Ortholengths and Hyperbolic Dehn Surgery, Ph.D. thesis, Univ. of Melbourne, Australia, 2000.

[9] D. B. A. Epstein and R. C. Penner, Euclidean decompositions of noncompact hyperbolic manifolds, J. Differential Geom. 27 (1988), no. 1, 67-80.

[10] W. Fenchel, Elementary geometry in hyperbolic space, de Gruyter, Berlin, 1989.

[11] W. M. Goldman, Geometric structures on manifolds and varieties of representations in Geometry of group representations (Boulder, CO, 1987), 169-198, Contemp. Math. 74, Amer. Math. Soc., Providence, RI, 1988.

[12] F. González-Acuña and J. M. Montesinos-Amilibia, On the character variety of group representations in $\mathrm{SL}(2, \mathbb{C})$ and $\operatorname{PSL}(2, \mathbb{C})$, Math. Z. 214 (1993), no. 4, $627-652$.

[13] O. A. Goodman and C. D. Hodgson, tube, 1999. This program is available from: http://www.ms. unimelb.edu.au/ ${ }^{\sim}$ snap

[14] J. Harris, Algebraic Geometry: A First Course, GTM 133, Springer, New York, 1992.

[15] H. Hilden, M. T. Lozano and J. M. Montesinos-Amilibia, On a remarkable polyhedron geometrizing the figure eight knot cone manifolds, J. Math. Sci. Univ. Tokyo 2 (1995), no. 3, 501-561.

[16] C. D. Hodgson, Degeneration and regeneration of geometric structures on 3 manifolds, Ph. D. thesis, Princeton Univ., 1986.

[17] C. D. Hodgson and S. Kerckhoff, Universal Bounds for Hyperbolic Dehn Surgery, in preparation.

[18] S. P. Kerckhoff, Deformations of hyperbolic cone-manifolds in Topology and Teichmüller spaces (Katinkulta, 1995), 101-114, World Sci. Publishing, River Edge, NJ, 1996.

[19] S. Kojima, Deformations of hyperbolic 3-cone-manifolds, J. Differential Geom. 49 (1998), no. 3, 469-516.

[20] S. Kojima, Hyperbolic 3-manifolds singular along knots, Chaos Solitons Fractals 9 (1998), no. 4-5, 765-777.

[21] G. R. Meyerhoff, The ortho-length spectrum for hyperbolic 3-manifolds, Quart. J. Math. Oxford Ser. (2) 47 (1996), no. 187, 349-359. 
[22] W. D. Neumann and D. Zagier, Volumes of hyperbolic three-manifolds, Topology 24 (1985), no. 3, 307-332.

[23] C. Petronio and J. Porti, Negatively oriented ideal triangulations and a proof of Thurston's hyperbolic Dehn filling theorem, Expo. Math. 18 (2000), no. 1, 1-35.

[24] D. Rolfsen, Knots and Links, Publish or Perish, 1976.

[25] W. P. Thurston, The geometry and topology of 3-manifolds, lecture notes, Princeton Univ., 1978. These notes are available from: http://msri.org/publications/books/gt3m/

[26] W. P. Thurston, Three-dimensional manifolds, Kleinian groups and hyperbolic geometry, Bull. Amer. Math. Soc. 6 (1982), 357-381.

[27] W. P. Thurston, Three-dimensional geometry and topology. Vol. 1, Princeton Univ. Press, Princeton, NJ, 1997.

[28] J. Weeks, SnapPea, the computer program, available from: http: //thames.northnet.org/weeks/index/SnapPea.html

Department of Mathematics, University of Melbourne Parkville, 3052, Australia

Email: jamesdowty@bigpond.com.au

Received: 24 October $2001 \quad$ Revised: 24 May 2002 